\title{
Solutions of the Two-Dimensional Hubbard Model: Benchmarks and Results from a Wide Range of Numerical Algorithms
}

J. P. F. LeBlanc, ${ }^{1}$ Andrey E. Antipov, ${ }^{1}$ Federico Becca, ${ }^{2}$ Ireneusz W. Bulik, ${ }^{3}$ Garnet Kin-Lic Chan ${ }^{4}$ Chia-Min Chung, ${ }^{5}$ Youjin Deng, ${ }^{6}$ Michel Ferrero, Thomas M. Henderson, ${ }^{3,8}$ Carlos A. Jiménez-Hoyos, ${ }^{3}$ E. Kozik, ${ }^{7}$ Xuan-Wen Liu, ${ }^{6}$ Andrew J. Millis, ${ }^{10}$ N. V. Prokof'ev, ${ }^{11,12}$ Mingpu Qin, ${ }^{13}$ Gustavo E. Scuseria, ${ }^{3,8}$ Hao Shi, ${ }^{13}$ B. V. Svistunov, ${ }^{11,12}$ Luca F. Tocchio, ${ }^{2}$ I. S. Tupitsyn, ${ }^{11}$ Steven R. White, ${ }^{5}$ Shiwei Zhang, ${ }^{13}$ Bo-Xiao Zheng, ${ }^{4}$ Zhenyue Zhu, ${ }^{5}$ and Emanuel Gull ${ }^{1, *}$

(Simons Collaboration on the Many-Electron Problem)

\author{
${ }^{1}$ Department of Physics, University of Michigan, Ann Arbor, Michigan 48109, USA \\ ${ }^{2}$ CNR-IOM-Democritos National Simulation Centre and International School for Advanced Studies \\ (SISSA), Via Bonomea 265, I-34136 Trieste, Italy \\ ${ }^{3}$ Department of Chemistry, Rice University, Houston, Texas 77005, USA \\ ${ }^{4}$ Department of Chemistry, Princeton University, Princeton, New Jersey 08544, USA \\ ${ }^{5}$ Department of Physics and Astronomy, University of California-Irvine, Irvine, California 92617, USA \\ ${ }^{6}$ Hefei National Laboratory for Physical Sciences at Microscale and Department of Modern Physics, \\ University of Science and Technology of China, Hefei, Anhui 230026, China \\ ${ }^{7}$ Centre de Physique Theorique, Ecole Polytechnique, CNRS, 91128 Palaiseau Cedex, France \\ ${ }^{8}$ Department of Physics and Astronomy, Rice University, Houston, Texas 77005, USA \\ ${ }^{9}$ Department of Physics, King's College London, Strand, London WC2R 2LS, United Kingdom \\ ${ }^{10}$ Department of Physics, Columbia University, New York, New York 10027, USA \\ ${ }^{11}$ Department of Physics, University of Massachusetts, Amherst, Massachusetts 01003, USA \\ ${ }^{12}$ Russian Research Center "Kurchatov Institute," 123182 Moscow, Russia \\ ${ }^{13}$ Department of Physics, College of William and Mary, Williamsburg, Virginia 23187, USA \\ (Received 9 May 2015; revised manuscript received 30 October 2015; published 14 December 2015)
}

Numerical results for ground-state and excited-state properties (energies, double occupancies, and Matsubara-axis self-energies) of the single-orbital Hubbard model on a two-dimensional square lattice are presented, in order to provide an assessment of our ability to compute accurate results in the thermodynamic limit. Many methods are employed, including auxiliary-field quantum Monte Carlo, bare and bold-line diagrammatic Monte Carlo, method of dual fermions, density matrix embedding theory, density matrix renormalization group, dynamical cluster approximation, diffusion Monte Carlo within a fixed-node approximation, unrestricted coupled cluster theory, and multireference projected Hartree-Fock methods. Comparison of results obtained by different methods allows for the identification of uncertainties and systematic errors. The importance of extrapolation to converged thermodynamic-limit values is emphasized. Cases where agreement between different methods is obtained establish benchmark results that may be useful in the validation of new approaches and the improvement of existing methods.

DOI: 10.1103/PhysRevX.5.041041

\section{INTRODUCTION}

The "many-electron problem" of providing a useful and sufficiently accurate calculation of the properties of

\footnotetext{
*egull@umich.edu
}

Published by the American Physical Society under the terms of the Creative Commons Attribution 3.0 License. Further distribution of this work must maintain attribution to the author(s) and the published article's title, journal citation, and DOI.
Subject Areas: Computational Physics,

Condensed Matter Physics,

Strongly Correlated Materials systems of large numbers of interacting electrons is one of the grand scientific challenges of the present day. Improved solutions are needed both for the practical problems of materials science and chemistry and for the basic science questions of determining the qualitative behaviors of interacting quantum systems.

While many problems of implementation arise, including calculation of the multiplicity of orbitals and interaction matrix elements needed to characterize real materials, the fundamental difficulties are that the dimension of the Hilbert space needed to describe an interacting electron 
system grows exponentially in the system size so that direct diagonalization is not practical except for small systems and that the minus sign associated with the Fermi statistics of electrons leads to exponentially slow convergence of straightforward Monte Carlo calculations. It is generally accepted that a complete solution to the many-electron problem cannot be obtained in polynomial time.

The difficulties associated with obtaining a complete solution have motivated the development over the years of approximate methods, and comparison of the different approximations remains a crucial open question. In this paper, we address this issue in the context of the repulsive-interaction Hubbard model [1-3] defined on a two-dimensional (2D) square lattice. The Hubbard model is one of the simplest models of interacting fermions, but despite its simplicity, it exhibits a wide range of correlated electron behavior including interaction-driven metal-insulator transitions, superconductivity, and magnetism. The precise behavior depends delicately on parameters, creating an interesting challenge for theory and computation.

Exact solutions are available for one-dimensional [4] and infinite-dimensional cases $[5,6]$. High-temperature series expansions provide numerically exact results but only for temperatures too high to be relevant for physically interesting situations [7]. In general dimensions at relevant temperatures, only approximate solutions are available. In some cases, these provide rigorous bounds to the groundstate energies or other thermodynamic properties [8,9]. Analytical perturbative methods can provide information about the behavior at very small interaction strengths [10-16] and at very large interaction strengths (for the special case of nearly one electron per site) $[17,18]$, but outside of these limits, obtaining results requires numerical methods [19]. Other techniques such as diagrammatic resummation are expected to work well in the weak coupling regime [20]. The known numerical methods are based on approximation schemes. Among the approximations employed are the study of finite systems (either directly or via embedding constructions), use of variational wave functions, and evaluation of subsets of all possible Feynman diagrams. Controlling these approximations and assessing the remaining uncertainties is a challenging but essential task, requiring analysis of results obtained from different methods. The past decade has seen the development of interesting new methods and substantial improvements in capabilities of previously developed approaches, suggesting that the time is ripe for a careful comparison.

In this paper, we undertake this needed task. Our aim is to assess the state of our knowledge of the Hubbard model, identifying parameter regimes where reliable results have been established and regimes where further work is needed. In regimes where reliable results have been established, our results will serve as benchmarks to aid in the development and validation of new methods.
This improved understanding of the Hubbard model will serve as a tool to analyze methods for solving the general many-electron problem.

We take the view that the only meaningful points of comparison between methods are results for the actual thermodynamic limit of the Hubbard model, with the uncertainties arising from the needed extrapolations (to infinite system size, to all diagrams, to infinite statistical precision in Monte Carlo calculations, etc.) quantified. However, examination of results obtained at different stages of the extrapolation sequence for a given method provide considerable information. Therefore, we present, when needed, both converged values and the intermediate results from which these were obtained.

The methods considered are auxiliary-field quantum Monte Carlo (AFQMC) [21-23], bare and bold-line diagrammatic Monte Carlo (DiagMC) [24-26], the dual fermion method (DF) [27], density matrix embedding theory (DMET) [28,29], density matrix renormalization group theory (DMRG) [30,31], cluster dynamical meanfield theory in the dynamical cluster approximation (DCA) [32], diffusion Monte Carlo based on a fixed-node approximation (FN) [33-39], unrestricted coupled cluster theory including singles and doubles (UCCSD), and in certain cases, higher excitations [40-42], and multireference projected Hartree-Fock (MRPHF) [43,44].

We examine energies and double occupancies, which are single numbers and can be obtained by essentially all methods, enabling straightforward comparison. We also consider properties related to the electron Green's function, which, at this stage, are only available from a few methods. The results obtained from different techniques enable us to identify regimes of phase space that are well understood, in the sense that several different methods provide results that are converged and agree within (reasonable) errors, and regimes that are not well understood, in the sense that there is as yet no agreement between different methods. We show excellent agreement and small uncertainties between numerically exact techniques at half filling (all coupling strengths), weak coupling (all carrier concentrations), and for carrier concentrations far from half filling (most interaction strengths). For carrier concentrations near half filling and for intermediate interaction strengths, results can be obtained, but the resulting uncertainties are much larger, in general, and more difficult to eliminate. We surmise that at least part of this uncertainty has a physical origin related to the presence of several competing phases, leading to sensitive dependence on parameters.

The rest of this paper is organized as follows. In Sec. II, we define the model, delineate parameter regimes, and define and discuss the observables of interest in this paper. In Sec. III, we summarize the methods, giving brief descriptions and focusing on issues most relevant to this paper while referring the reader to the literature for detailed descriptions. Section IVA demonstrates the importance of the extrapolation of results to the thermodynamic limit and 
TABLE I. Parameters studied for the Hubbard model. Here, $t$ denotes the nearest-neighbor hopping, $t^{\prime}$ the next-nearest-neighbor hopping, $U$ the interaction strength, $n$ the density, and $T$ the temperature.

\begin{tabular}{lccccc}
\hline \hline Parameters & \multicolumn{5}{c}{ Parameters studied } \\
\hline$t^{\prime} / t$ & -0.2 & 0 & 0.2 & & \\
$U / t$ & 2 & 4 & 6 & 8 & 12 \\
$n$ & 1.0 & 0.875 & 0.8 & 0.6 & 0.3 \\
$T / t$ & 0 & $1 / 8$ & $1 / 4$ & $1 / 2$ & \\
\hline \hline
\end{tabular}

discusses the issues involved in the extrapolations while Sec. IV B summarizes sources of uncertainty. In Secs. V, VI, and VII we present static observables in the strong coupling, intermediate coupling, and weak coupling regimes, respectively. Section VIII presents momentum and frequency dependence at finite $T$. A conclusion summarizes the work, outlines the important areas where our present-day knowledge is inadequate, and indicates directions for future research. Supplementary material presents the thermodynamic-limit values obtained here. A database of results is made available electronically $[45,46]$.

\section{THE HUBBARD MODEL}

The Hubbard model is defined by the Hamiltonian

$$
H=-\sum_{i, j, \sigma} t_{i j}\left(c_{i \sigma}^{\dagger} c_{j \sigma}+\text { H.c. }\right)+U \sum_{i} n_{i \uparrow} n_{i \downarrow},
$$

where $c_{i \sigma}^{\dagger}\left(c_{i \sigma}\right)$ creates (annihilates) an electron with spin $\sigma=\uparrow, \downarrow$ on site $i, n_{i \sigma}=c_{i \sigma}^{\dagger} c_{i \sigma}$ is the number operator, and $t_{i j}$ denotes the hopping term. In this work, we restrict our discussion to the repulsive Hubbard model $(U>0)$ defined on a two-dimensional square lattice; we further assume that the hopping contains only nearest-neighbor $\left(t_{i j}=t\right)$ and second-nearest-neighbor $\left(t_{i j}=t^{\prime}\right)$ terms. Here, $t$ is used to set the scale of all energies presented in this work.

We consider interaction strengths ranging from $U / t=2$ to $U / t=12$ and focus on temperatures where hightemperature expansion methods fail [7]. We give representative results for the ground state and temperatures $T / t=0.125,0.25$, and 0.5 . Table I contains a complete list of the parameters studied.

At zero temperature, we systematically compute the energy per site and the double occupancy, and we also present some data on the nature of the order and the order parameter, where an ordered phase is found. At nonzero temperature, we also present dynamical information, in particular, the Matsubara self-energy. We focus on values at a given density, rather than at a given chemical potential. This implies that methods based on a grand-canonical formulation need to adjust the chemical potential to find the right density, leading to additional uncertainty in computed quantities.

\section{METHODS}

\section{A. Overview}

Many numerical methods provide solutions to the Hubbard Hamiltonian on a finite-size lattice. In this work, we restrict attention to techniques that can access systems large enough that an extrapolation to the infinite system can be performed, and our aim is to obtain results for the thermodynamic limit. Also important to our analysis is the assessment of uncertainties, either by providing an unbiased error bar or an error bar that contains all errors except for a systematic contribution that may be assessed by comparison to other methods or reference systems.

We consider three broad classes of methods: ground-state wave function methods, embedding methods, and Green's function methods. The distinction between these methods is not sharp; several of the algorithms fit into multiple categories, but the categorization provides a useful way to organize a discussion of the different kinds of uncertainties.

Wave-function methods construct an approximation to the ground-state wave function of a system. Expectation values of observables (energies and correlation functions) are then evaluated by applying operators to this groundstate wave function. The issues are the accuracy of the wave function for a given system size and the accuracy of the extrapolation to the thermodynamic limit. AFQMC (with and without constrained path $C P$ ), UCCSD, FN, MRPHF, and DMRG are wave-function methods.

Embedding methods approximate properties of the full system (for example, the self-energy or density matrix) by the solution of a finite cluster self-consistently embedded in an appropriately designed infinite lattice. The full solution of the original problem is recovered as the cluster size is taken to infinity. Errors in embedding methods arise from three sources: the solution of the cluster problem, the convergence of the self-consistency loop that performs the embedding, and finite cluster size, with maximum cluster sizes depending on the method and ranging from 16 to 100. The DMET, DCA, and DF are embedding methods.

Green's function methods are defined here as methods based on stochastic evaluations of many-body perturbation series. They provide many-body self-energies $\Sigma\left(k, i \omega_{n}\right)$ and Green's functions $G\left(k, i \omega_{n}\right)$, as functions of momenta $k$ and fermionic Matsubara frequency $i \omega_{n}$, from which other observables (energies and densities) can be calculated. Techniques that produce real-frequency (rather than Matsubara frequency) information [47-50] are either restricted to small systems, molecules or impurity models, or work best at weak coupling. DiagMC is a Green's function method formulated directly in the infinite lattice; the main issues for this method are the accuracy of the stochastic approximation to the full diagrammatic expansion and the systematic truncation and extrapolation of the series. The DF and DCA techniques use Green's function techniques to evaluate the impurity problem and the expansion around it; 
they therefore are subject both to embedding uncertainties and to the uncertainties arising from the evaluation of the diagrammatic expansion.

\section{B. Auxiliary-field quantum Monte Carlo}

The AFQMC method is a ground-state wave-function method based on the idea that in the limit $\beta \rightarrow \infty$ the operator $e^{-\beta H}$ applied to an initial wave function $\left|\psi^{(\beta=0)}\right\rangle$ projects out the ground state of the Hamiltonian $H$. The projection is formulated as an imaginary-time path integral that is stochastically evaluated with the help of auxiliary fields introduced by a Hubbard-Stratonovich transformation. The method is applied to finite-size lattices, and an extrapolation to the infinite lattice case is required. If the calculation is converged, the exact ground-state energy and wave function for the lattice are obtained. The issues are the convergence of the stochastic evaluation of the projector and, when particle-hole symmetry is broken, the presence of a sign problem. The sign problem is managed using a constrained-path approximation, which introduces a potential systematic error that must be quantified by comparison to other methods. For an introduction to the basics of AFQMC methods, see, e.g., Ref. [21].

In this manuscript, we present results obtained from two ground-state AFQMC methods. The first [22] is based on the ground-state path integral form of AFQMC [51-53] but introduces several new algorithmic ingredients including an acceleration technique [54] (with force bias [21,55]) in the Metropolis sampling and control of Monte Carlo variance divergence [22]. This approach is applied to systems at half filling with $t^{\prime}=0$, where the sign problem [56] is absent because of particle-hole symmetry [57]. The algorithmic improvements allow us to reach longer imaginary time in the calculations, achieve a higher acceptance ratio, and greatly reduce the Monte Carlo variance [22]. The second approach we employ, to treat cases where the sign problem does occur, is referred to as the constrained-path Monte Carlo method [58,59]. This approach controls the sign problem with a constraint (implemented via a choice of trial wave function [21] $\left|\Psi_{T}\right\rangle$ ) on the paths in auxiliaryfield space, which allows stable calculations for arbitrarily long imaginary time and system size.

Both methods obtain the ground state of the Hamiltonian for a supercell of size $L \times L$ under twist-averaged boundary conditions $[60,61]$. The ground state is obtained by use of Monte Carlo methods to estimate $\left.\left|\psi^{(\beta)}\right\rangle=e^{-\beta H} \psi^{(\beta=0)}\right\rangle$. The total projection length is typically $\beta=64$ in the groundstate projection method, although test calculations with imaginary-time lengths several times larger were performed. The convergence error from finite values of $\beta$ is negligible. In the constrained-path method, the runs are open ended and tend to correspond to much larger values of $\beta$. In both the ground-state projection and constrained-path methods, the statistical error from the Monte Carlo calculation can be reliably estimated (1-standard-deviation error bars are reported). The systematic error from the constrained-path method is not variational but depends on $\left|\Psi_{T}\right\rangle$ in the sense that it vanishes if $\left|\Psi_{T}\right\rangle$ is exact. Its magnitude will be quantified below by comparison to other techniques.

A Trotter decomposition is used in the imaginary-time evolution. The Trotter error from the finite time step, $\Delta \tau=\beta / n$, must be extrapolated to zero. This extrapolation can be controlled and does not make a significant contribution to the error budget. Most calculations reported here use a time step fixed at $\Delta \tau=0.01$ in units of $t$.

Results obtained for finite $L$ are averaged over twist angle $\Theta$ to remove one-body finite-size effects. For small systems, a large number of $\Theta$ values are needed (about 200 for $4 \times 4$ or $6 \times 6$ ) $[59,61,62]$, but for larger systems, far fewer $\Theta$ are required to reach the same level of accuracy (for an $L \times L$ system with $L=20$, averaging over five twist angles is sufficient). These results are then extrapolated to $L \rightarrow \infty$. The extrapolation requires care because the ground state depends on the system geometry. For $n=0.875$, we used rectangular supercells (mostly $8 \times 32$, checked with sizes $8 \times 16,16 \times 16$, and $8 \times 48$ for consistency) to accommodate spin- and charge-density wave orders. The extrapolation also requires careful attention to the functional form of the leading finite volume correction [62-66]. Our final results at the thermodynamic limit include all statistical errors and a conservative estimate of the uncertainty resulting from the extrapolation of $L \rightarrow \infty$ in order to remove the two-body finite-size effects. (The fit includes $1 / L^{3}$ and $1 / L^{4}$ terms [61-63] for the energies and $1 / L$ for magnetization $m^{2}$.) To provide a concrete example, at $n=1, t^{\prime}=0, T=0, U / t=4$, the energy per site for a $20 \times 20$ system after $\Theta$ averaging is $E / t=-0.86038 \pm 3 \times 10^{-5}$. After a weighted leastsquare fitting with $L=4,6, \ldots, 18,20$, the final result in the thermodynamic limit is $E / t=-0.8603 \pm 2 \times 10^{-4}$.

For $n \neq 1$ or $t^{\prime} \neq 0$, a sign problem appears. The sign problem makes it impossible to converge the ground-state projection method for the system sizes and propagation lengths $\beta$ needed, and an alternative method, the $C P$ approximation, is used. The results reported in this paper follow Ref. [21], using a trial wave function $\left|\Psi_{T}\right\rangle$ to apply a boundary or gauge condition on the paths that are included in the path integral in auxiliary field space. All results reported here used single-determinant $\left|\Psi_{T}\right\rangle$ with no release. In these calculations, $\left|\Psi_{T}\right\rangle$ is taken to be a mean-field solution for the Hubbard model for given $U, L$, and $\Theta$ with a $U$ value $U_{\text {eff }}=\min \{U, 4 t\}$. The order parameter in the mean-field solution is chosen to be orthogonal to the spin quantization axis. This choice is found to help preserve symmetry in $\left|\Psi_{T}\right\rangle$, improving the $C P$ results $[23,67]$. The accuracy of the constrained-path approach has been extensively benchmarked $[23,61,62,67]$. We have carried out additional comparisons with exact diagonalization on $4 \times$ 4 systems. At $U=4 t$, the relative error on the energy, averaged over 60 randomly chosen $\Theta$ values, is $+0.018 \%$ for 
$n=0.25$ and $-0.15 \%$ for $n=0.625$. At $U / t=8$ and $n=0.875$, the relative error is $-0.51 \%$ averaged over 20 random $\Theta$ values. We have also verified these estimates in a few systems of larger $L$, using multideterminant trial wave functions and constraint release $[23,67]$.

\section{Fixed-node diffusion Monte Carlo with nodes from variational Monte Carlo}

The variational Monte Carlo method constructs a trial wave function that approximates the exact ground state of a correlated Hamiltonian [33-37]. The wave function depends on parameters that are optimized by minimizing the expectation value of the Hamiltonian, which requires a Monte Carlo sampling whenever the trial state is correlated (i.e., it is not a simple Slater determinant). We remark that the variational Monte Carlo energy gives an upper bound to the exact value and that, with this approach, it is possible to access quite large clusters, with all relevant spatial symmetries (translations, rotations, and reflections) preserved. However, it is difficult to quantify the systematic errors introduced by the choice of the trial state. Moreover, while spatial correlations may be correctly captured, dynamical properties are missed.

We generated simple variational wave functions by applying a density Jastrow factor on top of uncorrelated states that are built from local (mean-field) Hamiltonians, containing only a few parameters, where the physical properties are reflected in a transparent way as different terms inside the variational state $[68,69]$. At finite doping, the uncorrelated states have been obtained from the BardeenCopper-Schrieffer (BCS) Hamiltonian, including superconducting pairing on top of electron hopping; in addition, collinear antiferromagnetism with Néel order parallel to the $z$ spin quantization axis is also included. At half filling, where the system exhibits long-range magnetic order, the uncorrelated state contains only magnetism in the $x-y$ plane; in this case, an additional spin Jastrow factor involving the $z$ component of the spin operator is also taken. This term couples spins along a direction orthogonal to the magnetic ordering plane, reproducing the spin-wave fluctuations above the mean-field state [70]. All these variational wave functions with Jastrow factors generalize the so-called Gutzwiller state [2], allowing a description of metals, superconductors, and also Mott insulators [68,69]. Nevertheless, they do not give an accurate approximation to the exact ground state in two spatial dimensions, especially close to half filling. We obtain a substantial improvement by including the backflow correlations inside the uncorrelated state. On the lattice, this corresponds to a redefinition of the singleparticle orbitals, and it is particularly efficient at strong coupling [37,71].

To determine the variational parameters (i.e., the ones related to the Jastrow factors, the ones included in the meanfield Hamiltonian, and also those of the backflow correlations), we minimize the expectation value of the Hamiltonian. This minimization is performed by constructing a Markov chain using the Metropolis algorithm, where walkers are defined by many-body configurations having electrons on lattice sites with given spin along the $z$ axis. After performing this optimization, a further improvement can be obtained by applying the Green's function Monte Carlo projection technique [38] to the optimal trial state within a fixed-node approximation [39]. This procedure allows accurate calculations of the energy and diagonal correlation functions, such as double occupancies or density-density correlation functions. The Ansatz on the nodal structure given by the variational wave function induces a systematic error, which cannot be determined a priori but can be estimated from the change in energy as the trial wave function is improved. We point out that there is a difference between continuum and lattice fixed-node approaches. In the continuum, only the signs of the trial function are important: If the nodes are correctly placed, the exact energy is obtained. By contrast, on the lattice, both the signs and the relative magnitudes of the trial function in configurations that are connected by a sign flip must be correct in order to have the exact energy [39]. The error bars for finite systems are given as the statistical errors of the Green's function Monte Carlo technique and do not include any estimates of the systematic errors coming from the fixed-node approximation.

The finite-size results are then extrapolated to the infinite system size by using a scaling that depends on the carrier concentration. At half filling, we use the $1 / N^{3 / 2}$ scaling (where $N$ is the system size) that is appropriate for twodimensional ordered antiferromagnets $[64,65]$. In this regime, the error bar for the infinite system size is given by a fitting error of the linear regression. At finite dopings, the size scaling may suffer from shell effects: A smooth behavior can be obtained only when a sequence of closed-shell configurations are taken (i.e., electron fillings for which the noninteracting case corresponds to a unique ground state). In the generic case, size effects may be dominated by the ones present at $U=0$. This is the case for large dopings (e.g., $n=0.8)$ and all interactions, and intermediate dopings (e.g., $n=0.875)$ and small interactions $(U \lesssim 4)$. Here, for every available size, the ratio between the energy at finite $U$ and the one at $U=0$ is roughly constant and the thermodynamic limit can be assessed by fitting this ratio; namely, the infinite-size energy is obtained by multiplying the aforementioned ratio by the thermodynamic value at $U=0$. The extrapolated value is assumed to be normally distributed with an error bar taken as the difference between the estimated thermodynamic limit and the largest available size. For intermediate dopings $(n=0.875)$, the size scaling starts to deviate from the $U=0$ case around $U / t=4$, and we decided to take the point at the largest size as the infinite size limit, with an uncertainty of twice the difference to the next-lowest system size. We remark that, in this case, a linear regression with the $1 / N$ scaling gives an estimate of the thermodynamic limit that is compatible (within one error bar) with the point at the largest size. 


\section{Multireference projected Hartree-Fock method}

The multireference projected Hartree-Fock method $[43,44,72]$ is a ground-state wave-function approach based on a trial wave function $|\Psi\rangle$ characterized by the quantum numbers $\Theta, K$ that is constructed out of a set of brokensymmetry Hartree-Fock wave functions $\left(\left\{\left|\mathcal{D}_{i}\right\rangle\right\}\right)$ via projection operators. The idea is that a broken-symmetry determinant includes the dominant correlation physics, while the projection restores the physical symmetries. The wave function takes the form

$$
\left|\Psi_{K}^{\Theta}\right\rangle=\sum_{i=1}^{n} \sum_{K^{\prime}} f_{K^{\prime}}^{i, \Theta} \hat{P}_{K K^{\prime}}^{\Theta}\left|\mathcal{D}^{i}\right\rangle
$$

and the parameters $f_{K^{\prime}}^{i, \Theta}$ are determined by minimizing the energy. The projector $\hat{P}_{K K^{\prime}}^{\Theta}$ restores the symmetries (characterized by the quantum numbers $\Theta, K)$ in $|\Psi\rangle$ and can be formally written as $[73,74]$

$$
\hat{P}_{K K^{\prime}}^{\Theta}=\frac{h}{L} \sum_{m} \Gamma_{K K^{\prime}}^{\Theta *}(m) \hat{R}(m)
$$

in terms of the rotation operators $\hat{R}(m)$ and the irreducible representation matrices $\Gamma^{\Theta}(m)$ associated with the elements $m$ of the symmetry group of the problem. Here, $h$ is the dimension of the irreducible representation, while $L$ is the volume of the group. The character of the brokensymmetry determinant is optimized in the presence of the projection operator (i.e., a variation-after-projection approach), which results in broken-symmetry determinants with well-defined defects [44].

Our expansion employs Slater determinants that break the space group and spin $\left(\hat{S}^{2}\right)$ symmetries of the lattice but preserve $S_{z}$ symmetry. All the broken symmetries are restored using the appropriate projection operators. The series of $i$ determinants in Eq. (2) is constructed through a chain of variational calculations, using the few-determinant (FED) approach $[43,44,74]$. In this procedure, after a wave function with $n-1$ intrinsic determinants is available, a wave function with $n$ determinants is variationally optimized by adjusting the Thouless coefficients determining the last-added determinant. The full set of linear coefficients $f_{K^{\prime}}^{i \Theta}$ is readjusted. The MRPHF approach becomes exact as the number of determinants retained tends to the size of the Hilbert space, i.e., as Eq. (2) becomes a coherent-state representation of the exact ground-state wave function.

If the number of determinants is fixed at a finite, not-toolarge value, these calculations can be performed for large lattices with a polynomial cost $\left[\mathrm{O}(N)^{4}\right.$ or so, where $N$ is the number of sites in the lattice]. However, the number of determinants required to obtain results with a given accuracy increases exponentially with increasing system size. In this work, implementation aspects have compelled us to keep the number of determinants roughly constant, so the quality of the solution decreases as the system size is increased, and, consequently, the energy increases, precluding a thermodynamic-limit extrapolation. We have used expansions with 4, 24, and 32 determinants for half-filled $4 \times 4,6 \times 6$, and $8 \times 8$ lattices, respectively. In the lightly doped regime, larger expansions have been used: 48 determinants in a $10 \times 4(\langle n\rangle=0.8)$ lattice and 80 determinants in a $16 \times 4(\langle n\rangle=0.875)$ one. A linear extrapolation in the reciprocal of the number of determinants, i.e., in $1 / n$, has been performed to the infinite configuration limit for the ground-state energies.

The calculations presented could, in principle, be improved in a number of ways: Additional symmetries could be broken and restored (such as $\hat{S}_{z}$ or particle number) in the reference configurations, more configurations could be included, and/or a full optimization of all determinants could be performed, in the spirit of the resonating Hartree-Fock approach [75]. The accuracy of any one result can therefore be increased, as shown by Rodríguez-Guzmán et al. [76] or by Mizusaki and Imada [77] in the closely related path integral renormalization group (PIRG) approach. Recently, Tahara and Imada [78] have combined the symmetry-projected determinant expansion with short- and long-range Jastrow factors within a variational Monte Carlo framework, which may be used to further increase the accuracy. These techniques, and others, have been used to explore, for example, spin and charge stripe phases, which we do not explore in this work [79-81].

\section{E. Unrestricted coupled cluster-singles and doubles}

Coupled cluster (CC) theory [40-42] is a ground-state wave-function technique. It is widely used in quantum chemistry and often considered the best source of precise data for molecules that are neither too large nor too strongly correlated. Its application to the Hubbard model has been more limited, where it has been used in two different formulations. In the first form, which directly exploits the translational invariance of the lattice to work in the thermodynamic limit, the theory is formulated in the site basis, starting from an infinite Néel-ordered reference, from which clusters of excitations that change occupancy and flip spins are created $[82,83]$. In the second form, the theory starts from a single-determinant reference state on a finite lattice, from which clusters of particle-hole excitations are created [84]. This is similar to how the theory is used in quantum chemistry and is the formulation discussed further here. As this second form does not work in the thermodynamic limit, the energies must be extrapolated.

The $\mathrm{CC}$ wave function is written as $|\Psi\rangle=\exp (\mathrm{T})|0\rangle$, where $|0\rangle$ is a single-determinant reference state, and $T=\sum_{n} T_{n}$, where $T_{n}=\sum_{q_{n}} t_{q_{n}} A_{q_{n}}^{\dagger}$ is the cluster operator. The operator $A_{q_{n}}^{\dagger}$ creates an excited determinant $\left|\Phi_{q_{n}}\right\rangle$ which contains $n$ particle-hole pairs relative to the reference 
state. In its standard and simplest version (used here), the energy and coefficients $t_{q_{n}}$ are obtained by solving the Schrödinger equation projectively:

$$
\begin{aligned}
& E=\left\langle 0\left|\mathrm{e}^{-T} H \mathrm{e}^{T}\right| 0\right\rangle, \\
& 0=\left\langle\Phi_{q_{n}}\left|\mathrm{e}^{-T} H \mathrm{e}^{T}\right| 0\right\rangle \quad \forall q_{n} .
\end{aligned}
$$

CC theory thus diagonalizes a similarity-transformed Hamiltonian $\bar{H}=\exp (-T) H \exp (T)$ in a subspace of states defined by $|0\rangle$ and $\left|\Phi_{q_{n}}\right\rangle$. Note that because $T$ is a pure excitation operator, the commutator expansion used to evaluate $\bar{H}$ truncates after four commutators.

If the sum over $n$ in defining the cluster operator is carried out to all orders, the exact ground-state wave function is reproduced. In practice, the operator $T$ is typically restricted to terms involving a small number of particle-hole pair excitations above the reference state $\left(n \leq n_{\max }\right)$, and $|\Psi\rangle=\exp (\mathrm{T})|0\rangle$ is projected onto the space of states with up to $n_{\max }$ particle-hole pairs; the accuracy then depends upon $n_{\max }$ and the choice of reference state $|0\rangle$. In a lattice model such as the Hubbard model with $N$ sites, the computational cost is, roughly speaking, proportional to $N^{2\left(n_{\max }+1\right)}$.

The calculations reported in this manuscript primarily limit $T$ to $n \leq 2$, i.e., to the creation of only singly and doubly excited determinants, giving what we refer to as CC with single and double excitations (CCSD) $[85,86]$. For select examples, we have included corrections for triple and occasionally quadruple excitation effects. The accuracy of $\mathrm{CC}$ theory, and the need for higher excitations, depends on how well the reference $|0\rangle$ captures the qualitative physics. When the reference is accurate, then single and double excitations may be sufficient; however, when the reference determinant bears little resemblance to the exact wave function (as may happen in strongly correlated systems), a much higher degree of excitation is required to recover the correct physics. For this reason, the calculations reported here use a symmetry-broken unrestricted Hartree-Fock (UHF) reference determinant because UHF can provide a better mean-field description, particularly near half filling where antiferromagnetic correlations dominate; this defines the UCCSD method used here. Note, however, that particularly for doped systems with large $U$, there are a plethora of nearly degenerate UHF states and finding the best reference for UCCSD is not straightforward. We have prepared the UHF solution following the prescription of Ref. [87]. In principle, the deficiencies of the reference determinant can be corrected in what is known as Brueckner CC [88], where the reference determinant is adjusted to eliminate single excitation effects. These calculations are more computationally demanding, and we have not pursued them here.

An important virtue of the exponential parametrization of the wave function is that the CC energy has a nontrivial thermodynamic limit even for restricted excitations $n_{\max }$ [40-42]. Thus, as the lattice size increases, the energy per site approaches the thermodynamic limit for the given $n_{\max }$ and the exact thermodynamic limit as $n_{\max }$ is increased. For smaller values of $U$ where convergence to the thermodynamic limit is slower, we have converged second-order perturbation theory out to the thermodynamic limit and added a correction for the difference between $\mathrm{CC}$ theory and perturbation theory, which, for small $U$, converges quickly with respect to the system size. Double occupancies have been computed by numerical differentiation of the CC energy with respect to $U$. We also provide UCCSDTQ estimates of the ground-state energy (labeled as UCCSDTQ $*$ ) for $n=1$ systems, where the triples (T) correction is obtained as UCCSDT-UCCSD energies for a $6 \times 6$ system, and the quadruples $(\mathrm{Q})$ correction is obtained from UCCSDTQ-UCCSDT energies for a $4 \times 4$ lattice. For $n=0.8(n=0.875)$, our UCCSDT estimates (labeled as UCCSDT*) are obtained from $10 \times 4(16 \times 4)$ lattices.

The UCCSD calculations reported here can be completed in a few hours using standard quantum chemistry packages [89]. Even at half filling where there are not many Hartree-Fock solutions to be concerned with, we find large effects due to single excitations, which suggests that the coupled cluster calculations could be substantially improved by optimizing the identity of the reference determinant. Similarly, we generally see significant corrections due to triple and higher excitations; these can also be computed with standard packages [90]. However, while optimizing the reference determinant and including higher excitation effects will increase the accuracy of the coupled cluster calculations, they also increase the cost.

\section{F. Density matrix renormalization group}

The density matrix renormalization group [30] is a variational ground-state wave-function technique. It constructs the ground state of a system by diagonalizing the Hamiltonian in a finite subspace spanned by an iteratively constructed basis that is optimized via a Schmidt decomposition that minimizes the spatial extent of the quantummechanical entanglement between basis states.

DMRG is generally believed to be the optimal method for finding ground states of one-dimensional lattice models. In the application to one-dimensional systems, two sources of error must, in principle, be controlled. Results for a fixed system length of $L$ must be converged with respect to basis size $m$, and then the converged results must be extrapolated to $L \rightarrow \infty$. However, for most of the one-dimensional Hamiltonians of current interest, the convergence is very rapid; in practice, large enough $m$ and $L$ are accessible numerically, so extrapolation is not required.

Application of DMRG to a finite-size 2D system proceeds by defining an effective one-dimensional problem, to which the standard one-dimensional DMRG is applied. Two-dimensional tensor network generalizations 
of the DMRG ideas have attracted tremendous interest recently, but these methods have not yet produced results for the two-dimensional Hubbard model that can be included in the present comparison [91,92].

Most current implementations of DMRG require open boundary conditions. The canonical method for creating an effective one-dimensional system from a finite-size twodimensional one is to impose periodic boundary conditions in one direction and open boundary conditions in the other, thereby defining a cylinder of finite length and finite circumference. One then defines an effective onedimensional problem by indexing the sites along a onedimensional path that covers all of the sites on the cylinder and taking the matrix elements of the Hamiltonian in this basis. The price is that two sites separated by a small distance along the cylinder axis in the physical system are separated by a distance of order the cylinder circumference in the effective one-dimensional model. The effective onedimensional problem thus has long-ranged terms, which imply longer-ranged entanglement and require that more states are kept in the optimal basis. The number of states needed grows exponentially with the circumference of the cylinder (width of the original finite lattice), meaning that there is a sharp cutoff in the accessible system widths, typically around width 6 in the Hubbard systems; however, systems of very large cylinder length can be studied. The extrapolation to the thermodynamic limit must thus be handled with care.

The DMRG calculations reported here were performed with the standard DMRG finite-system algorithm [30,31]. Two types of cylinders were considered: one with the axial and circumferential directions aligned parallel to the bonds of the square lattice and one rotated by $45^{\circ}$. When one cuts a cylinder in two, the $45^{\circ}$ rotated system has fewer sites on the boundary per unit length, and thus one expects a smaller growth of the entanglement with the length of the cut (governed by the area law). For the undoped antiferromagnetic system, the rotated system is also unfrustrated, both for odd and even circumferences, reducing shell effects in the finite-size results. For half-filled systems, both types of orientations are considered, and they show good agreement; error bars are estimated to incorporate both the error bars on the data points for specific widths and the differences between the two orientations. However, we note that for the half-filled systems, better results were obtained with the rotated system $[93,94]$. With doped systems, we see striping behavior at stronger coupling. A stripe is a line of holes that act as a domain wall in the antiferromagnetism on either side. These stripes have been seen in the t-J model with DMRG starting in the late 1990s [66]. The stripes typically wrap around the cylinder, with a specific even number of holes in the ring stripe. With doping, the optimal number of holes can change. Striped configurations with the wrong number of holes in a stripe are typically metastable. For the ordinary orientation, we were able to sort out stripe fillings, finding the low-energy states and avoiding metastability. For the $45^{\circ}$ rotated lattices, the patterns seem more delicate, and we have not yet sorted out the lowest-energy configurations. We thus present only the results for standard orientation for doped systems.

Convergence issues pose more severe problems than in the standard one-dimensional cases, both because of the intrinsic limits on system size discussed above and because, in some cases, the presence of several metastable states can cause trouble for extrapolation and can lead to the appearance of states that are not the ground state and may be important only for finite systems. In the systems studied here, metastability was traced to the presence of physically different "striped" states for different hole doping in the DMRG cylinder.

We obtain converged results as follows. For each cylinder of a specific length $L$, we extrapolate the energy in the number of basis states, $m$. To make this extrapolation reliable, $m$ is slowly increased, but each $m$ is used for two consecutive sweeps. The truncation error and the energy are measured on the second sweep for each $m$. Then, a linear extrapolation of energy versus truncation error is used to obtain the ground-state energy with error bars. The deterministic nature of DMRG can result in uncertainties due to fitting which do not appropriately represent the uncertainty in the choice of extrapolation procedure. We therefore assume a normally distributed error of $1 / 5$ the difference between the last point and the extrapolated value, which we justify by comparison to the accuracy of previous DMRG data $[93,94]$. Metastability is signaled by lack of linearity in this extrapolation. When this is found, we determine which state had the lowest energy. The system is then rerun with an initial state favoring the lower-energy state producing results in the lower-energy configuration such that the extrapolations are linear in the truncation error.

For a fixed width, we then extrapolate the energies linearly in $1 / L$ to get an energy per site for an infinite cylinder. Errors are estimated statistically using the error bars on each point. To reduce the finite-size effects from different widths, we employ a simple version of the phaseaveraging trick [60] by taking an average over periodic and antiperiodic boundary conditions around the cylinder. The phase averaging eliminates an oscillation in the energy as a function of system width in the $45^{\circ}$ rotated systems and, in general, provides a nicely accelerated convergence. We then analyzed the results as a function of cylinder width. For half filling, we found that extrapolating the energy to the thermodynamic limit by $1 /$ width $^{3}$ works well. This is the finite-size behavior in the Heisenberg model, where it is well understood [95].

\section{G. Density matrix embedding theory}

The DMET $[28,29]$ is a ground-state embedding method formulated in terms of wave-function entanglement. Given 
an impurity cluster of size $N_{c}$, DMET maps an $L \times L$ bulk many-body problem ( $L$ is chosen to be very large) to an impurity plus bath problem with $N_{c}$ correlated (impurity) sites and the same number of bath orbitals. The mapping is constructed from the Schmidt decomposition [96] of a bulk wave function $|\Psi\rangle$. The formulation is exact if $|\Psi\rangle$ is the exact bulk ground state, or in the limit of impurity cluster size $N_{c} \rightarrow \infty$. Since the exact bulk state is not known a priori, an approximate bulk state is used for the impurity mapping. Recently, DMET has been applied to both ground-state and linear response spectral properties of the Hubbard model [28,97-99]. In this work, we use a general BCS bulk state, the ground state of the DMET lattice Hamiltonian given by the hopping part of the Hubbard Hamiltonian augmented with the DMET correlation potential $u$, which is applied in each cluster supercell of $N_{c}$ sites in the bulk lattice. This bulk state is allowed to spontaneously break spin and particle-number symmetry through the self-consistency cycle that determines $u$ (which contains both particle-number conserving and nonconserving terms). In this cycle, the bulk state $|\Psi\rangle$ is updated from the interacting impurity and bath solution $|\Phi\rangle$, by minimizing the difference between $|\Psi\rangle$ and $|\Phi\rangle$ [as measured by their (generalized) one-particle density matrices] with respect to the potential $u$.

For the bulk lattice, we use $L=72$. From calculations on larger $L$, we find that the finite lattice error associated with this choice is negligible, on the scale of the significant digits reported. The BCS bulk state is obtained by solving the lattice spin-unrestricted Bogoliubov-de Gennes equation $[100,101]$ with the correlation potential $u$. The impurity and bath problem is solved in the BCS quasiparticle basis, with general one-body and two-body interactions that do not conserve particle number or locality. We use the DMRG [30] as an impurity solver (adapted from the quantum chemistry DMRG code BLOCK [102-104]), with a maximum number of renormalized states $m=2000$ (DMET self-consistency is performed up to $m=1200$ ). The DMET lattice and impurity Hamiltonians are augmented with a chemical potential $\mu$, to ensure that the relative error in particle number is less than $0.05 \%$. We use impurity clusters of dimensions $2 \times 2,4 \times 2$, $4 \times 4$, and $8 \times 2$ at each point in the phase diagram. The energies and observables are then extrapolated to the thermodynamic limit using a linear relationship with $N_{c}^{-1 / 2}$, as appropriate for a nontranslationally invariant cluster embedding theory.

The total DMET uncertainty is estimated by combining the errors from three sources: (i) convergence of DMET self-consistency, (ii) solution of the impurity many-body problem using DMRG, and (iii) extrapolation to the limit of infinite impurity size. We estimate the self-consistency error (i) using the difference of the last two DMET selfconsistent iterations. The average self-consistency error is below $5 \times 10^{-4} t$ in the energy for all cluster sizes. The impurity solver error (ii) is from using a finite number of renormalized states $m$ in DMRG. This error is only nonzero in clusters larger than $2 \times 2$. The energy and observables are extrapolated to $m=\infty$ using the standard linear relation between energy and DMRG truncation error $[93,102,105]$. For $4 \times 4$ impurity clusters, the truncation error is large enough to also contribute to the converged DMET self-consistent correlation potential $u(m)$. To take this into account, we extrapolate using (a) self-consistent DMET results converged at different $m$, and (b) non-selfconsistent DMET results using different $m$ in the DMRG impurity solver at a fixed correlation potential $u\left(m_{\max }\right)$, where $m_{\max }$ is the maximum $m$ used in the DMET selfconsistency. The difference between the two extrapolations is then added to the total DMET uncertainty. For the cluster sizes in this study, the errors due to (i) and (ii) are small and easily controlled. Therefore, the finite-size impurity error is the main source of uncertainty at almost all points in the phase diagram. It is estimated as the standard deviation of the finite-size extrapolation. The quality of the approximate DMET impurity mapping depends on the approximate lattice wave function $|\Psi\rangle$ and decreases as the coupling strength $U$ increases, especially for carrier concentrations near half filling. This slows down the cluster-size convergence of the results for large $U$, increasing the uncertainty. In the strong coupling, weakly doped region, we find competing homogeneous and inhomogeneous orders that become very sensitive to the cluster size and shapes (similar to the stripes observed in DMRG). It is difficult to reliably extrapolate these results to the thermodynamic limit. As a result, the total DMET uncertainties range from about $10^{-4} t$ at half filling and low densities to a maximum of about $10^{-2} t$ in the strong coupling, underdoped region. A detailed description of the methodology and extrapolation procedures for the calculations is contained in Ref. [106].

\section{H. Dynamical cluster approximation}

The DCA $[32,107,108]$ is an embedding technique in which an approximation to the electron self-energy is obtained from the solution of a quantum impurity model consisting of some number $N_{c}$ of interacting sites coupled to an infinite bath of noninteracting electrons. In applications to the Hubbard model, the interactions in the impurity model are the interactions of the original problem, while the one-body terms are determined from a self-consistency condition relating the Green's functions of the impurity model to those of the lattice. The DCA is a particular generalization to $N_{c}>1$ of the "single-site" dynamical mean-field method $[109,110]$. Other generalizations have also been introduced [111,112], but results from these methods are not considered here. The single-site method was motivated by the observation that in an appropriately defined infinite coordination number limit, an exact solution of the Hubbard model can be found [5] and can be recast in terms of the solution of a single-site impurity model [109]. It was later understood that generalization to 
$N_{c}>1$ impurity-model representations allows a treatment of the finite coordination number model that becomes exact as the number of impurity sites $N_{c} \rightarrow \infty$.

The DCA formulation partitions the Brillouin zone into $N_{c}$ equal area tiles and approximates the self-energy $\Sigma$ as a piecewise constant function of momentum taking a different value in each tile:

$$
\Sigma(\mathbf{k}, \omega)=\sum_{a=1 \ldots N_{c}} \phi_{a}(\mathbf{k}) \Sigma_{a}(\omega),
$$

with $\phi_{a}(\mathbf{k})=1$ for $\mathbf{k} \in a$ and $\phi_{a}(\mathbf{k})=0$ for $\mathbf{k} \notin a$. The tiles $a$ map directly onto impurity-model single-particle levels, the impurity-model Green's function and self-energy are diagonal in the impurity model $a$ basis, and the DCA self-consistency equation

$$
G_{a}^{\mathrm{imp}}(\omega)=\int_{a} \frac{d^{2} k}{(2 \pi)^{2}} \frac{1}{\omega-\varepsilon_{k}-\Sigma_{a}(\omega)}
$$

determines the remaining parameters of the impurity model. The self-consistency loop is solved by iteration; an initial guess for the impurity-model parameters produces a set of $\Sigma_{a}$, which are then used to update the impuritymodel parameters. The loop typically converges well, and errors associated with the self-consistency are smaller than errors in the solution of the impurity model.

We obtain results for different $N_{c}$ in the paramagnetic phase and extrapolate to the thermodynamic limit by exploiting the known [32] $N_{c}^{-d / 2}$ scaling for momentumaveraged quantities in $d$ dimensions and systematically increasing $N_{c}[113,114]$.

To solve the $N_{c}$-site impurity problem, we use a CTAUX algorithm [115,116] with submatrix updates [117]. Our codes are based on the ALPS libraries [118,119]. Selected points have been compared to a CT-INT [120] implementation based on the TRIQS libraries [121].

In this work, we provide extrapolated DCA results from clusters of sizes $N_{c}=16,20,32,34,50,64,72$, and 98, depending on temperature and densities, in order to give a reliable estimate of the properties of the 2D Hubbard model in the thermodynamic limit.

The CT-AUX method is a type of diagrammatic Monte Carlo method. In the $T>0$, impurity-model context, the diagrammatic series is absolutely convergent, and the issues discussed below for Diag-MC in the infinitelattice context are not important. However, the CT-AUX method is restricted at low $T$ by the existence of a Monte Carlo sign problem in the auxiliary field solver. The sign problem worsens rapidly as $U$ is increased, as $T$ is decreased, or as $N_{c}$ is increased, and this is particularly evident in the density range $n=[0.8,1.0)$. Furthermore, as temperature is decreased, the length scale of correlations in the system increases, resulting in larger finite-size effects. We take a conservative approach to determining the uncertainty in the extrapolation procedure. We include both a statistical uncertainty in the extrapolation in $1 / N_{c}$ and an additional uncertainty, which we take as half the difference of the extrapolated value from the largest $N_{c}$ value explored. This gives a robust representation of an extrapolation error, which is larger when finite-size effects are large but that also vanishes as $N_{c}$ increases. As such, the error bars for extrapolation of our DCA results to the thermodynamic limit contain both stochastic and finite-size uncertainties, and values for finite system sizes with stochastic error bars are provided.

\section{Dual fermion ladder approximation}

The dual fermion approach $[27,122,123]$ is a diagrammatic extension of the single-site dynamical mean-field theory (DMFT). The DF technique is motivated by the idea that nonlocal corrections to DMFT can be captured by a perturbative expansion around a solution of the dynamical mean-field equations. In formal terms, the expansion requires summing a series of diagrams for two and higher particle correlations, with vertices defined in terms of the fully interacting but reducible vertices of the impurity model. In this regard, the DF technique is similar in spirit to methods such as the dynamical vertex approximation $(D \Gamma A)$ and dynamical mean-field extensions of fRG $\left(\mathrm{DMF}^{2} \mathrm{RG}\right)$, which approximate interactions on the level of two-particle vertex functions [124-126]. In practical implementations to date, the dual fermion expansion is truncated at the two-particle level (higher than two-particle interactions are omitted), and the series of two-particle corrections is approximated by a few low-order terms or a ladder resummation. One of its strengths lies in the ability to describe phase transitions of the system by employing resummations of the relevant diagrams $[127,128]$.

The DF results presented here are obtained using the open-source opendf code [129], starting from a singlesite $\left(N_{c}=1\right)$ dynamical mean-field solution obtained with a continuous-time auxiliary-field (CT-AUX) [115-117] impurity solver. The method is limited by the accuracy to which reducible impurity vertex functions can be obtained, which is a polynomial (cubic) complexity problem. Within the approximation of neglecting higher-order vertices and only considering ladder diagrams, a systematic estimation of deviation from the true interacting system is not possible, and we omit error bars altogether.

\section{J. Diagrammatic Monte Carlo method}

The diagrammatic Monte Carlo method (DiagMC) begins from the observation that within standard manybody perturbation theory, any quantity $Q$ that depends on some set of arguments $\mathbf{y}$ (which may include both continuous components such as frequency and momentum and discrete components such as spin) may be expressed as an infinite series of terms, each of which involves multidimensional integrals and sums over many variables: 


$$
Q(\mathbf{y})=\sum_{\alpha=0}^{\infty} \sum_{\xi} \int \ldots \int d \mathbf{x}_{1} \ldots d \mathbf{x}_{\alpha} D\left(\alpha, \xi, \mathbf{x}_{1}, \ldots, \mathbf{x}_{\alpha} ; \mathbf{y}\right) .
$$

Here, the $D$ are known functions defined by the Feynman rules of diagrammatic perturbation theory. The series order $\alpha$ controls the number of internal integration or summation variables $\left\{\mathbf{x}_{1}, \ldots, \mathbf{x}_{\alpha}\right\}$, and $\xi$ labels different terms of the same order in the series. The quantity $Q$ may be the electron Green's function $G$, the self-energy $\Sigma$, the screened interaction $W$, the polarization operator $\Pi$, the pair propagator (for contact interaction) $\Gamma$ and its self-energy $\Sigma_{\Gamma}$, or the current-current or other correlation functions. Basic thermodynamic properties (particle, kinetic, and potential energy densities) in the grand canonical ensemble are readily obtained from $G$ and $\Sigma$ (see Ref. [130]).

The most widely used formulation of perturbation theory is in terms of Feynman diagrams. In this case, standard rules relate the graphical representation of a given term in the series to the corresponding mathematical expressions for the $D$, which are typically given (up to a sign or phase factor) by a product of functions associated with graph lines, $D=\prod_{\text {lines }} F_{\text {line }}\left(\mathbf{x}_{\text {line }}\right)$. In perturbation theory for particles interacting via pairwise forces, these lines are associated with the interaction potential $U$ and bare singleparticle propagators $G^{(0)}$. If we denote the collection of all external and internal variables that allow for a complete characterization of the diagram (diagram topology and internal and external variables) as $\nu \equiv\left(\alpha, \xi, \mathbf{x}_{1}, \ldots, \mathbf{x}_{\alpha} ; \mathbf{y}\right)$, then Eq. (7) can be viewed as the weighted average over the configuration space $\{\nu\}$ : i.e., $Q=\sum_{\nu} D_{\nu} \equiv \sum_{\nu} e^{i \varphi_{\nu}}\left|D_{\nu}\right|$, where the modulus of $D_{\nu}$ defines the configuration "weight," and $\varphi_{\nu}=\arg D_{\nu}$. The basic idea of the DiagMC method is to use stochastic sampling of the configuration space to compute $Q$ by treating $\propto\left|D_{\nu}\right|$ as the probability density for the contribution of point $\nu$ to the sum. In DiagMC, the diagram order, its topology, and all internal and external variables are treated on equal footing, and each diagram represents a point, not an integral, in $\{\nu\}$. The MC process of generating diagrams with probabilities proportional to their weight is based on the conventional Markov-chain updating scheme [24-26] implemented directly in the space of continuous variables.

Since connected Feynman diagrams are formulated directly in the asymptotic limit, there is no infinitesystem-size limit to take. The main numerical issue concerns the convergence of the Monte Carlo process, which is complicated by the exponential proliferation of the number of diagrams with perturbation order $\alpha$. This leads to exponential computational complexity since final results are recovered only after extrapolation to the infinite diagram-order limit. The fermion sign enters the calculation in an interesting way: Different diagrams have different signs (arising from the different orderings of fermion creation and annihilation operators), and at large diagram order, the contributions of diagrams with plus and minus signs tend to cancel. This cancellation is, in fact, responsible for the convergence of the many-body perturbation theory [26,131]. To manage this issue, it is useful to consider a Monte Carlo process for an approximate series in which the maximum diagram order is limited to some finite value via a hard or soft cutoff, and then the cutoff is increased until convergence is reached.

Diagrammatic Monte Carlo techniques can also take advantage of known field-theoretical techniques to run the calculation in a self-consistent mode in which certain infinite series of diagrams are summed and then automatically absorbed into the renormalized propagators and interaction lines using Dyson-type equations. One example is the skeleton expansion [131]; another is a "bold" expansion in perturbative corrections to an analytic partial resummation [132,133]. This flexibility allows for an additional control over systematic errors coming from series extrapolation as well as convergence issuesdifferent schemes should produce consistent final results.

In this work, we employ four complementary techniques:

(i) $\mathrm{A}\left[G^{(0)}\right]^{2} U$ scheme based on a Taylor series expansion of $\Sigma$ in powers of $U$ with fixed shifted chemical potential $\tilde{\mu}=\mu-U n / 2$ (see Refs. [26,134]). The total electron density $n=\sum_{\sigma} n_{\sigma}$ and the chemical potential are computed a posteriori (after results are extrapolated to the $\alpha_{\max } \rightarrow \infty$ limit).

(ii) $\mathrm{A} G^{2} W$ scheme based on skeleton series for $\Sigma$ and $\Pi$ in which all lines in the diagram are understood as fully dressed Green's functions and screened interactions. Self-consistency is implemented by feedback loops when $G$ and $W$ are obtained by solving algebraic Dyson equations, $G^{-1}=\left[G^{(0)}\right]^{-1}-\Sigma$ and $W^{-1}=U^{-1}-\Pi$, in momentum-frequency representation (see Refs. [135-137] for more details).

(iii) $\mathrm{A} G^{2} \Gamma$ scheme based on the skeleton series for $\Sigma$ and $\Sigma_{\Gamma}$ when all lines in the graph are understood as fully dressed single-particle (Green's functions) and twoparticle propagators. This compact formulation is possible only for a contact interaction potential when the sum of ladder-type diagrams for spin-up and spindown particles has the same functional structure as the single-particle propagator (see Refs. [132,138,139]). Again, self-consistency is implemented by feedback loops using Dyson equations, $G^{-1}=\left[G^{(0)}\right]^{-1}-\Sigma$ and $\Gamma^{-1}=\left[\Gamma^{(0)}\right]^{-1}-\Sigma_{\Gamma}$, where $\Gamma^{(0)}$ is the sum of bare ladder diagrams.

(iv) $\mathrm{A}\left[G^{(0)}\right]^{2} \Gamma^{(0)}$ scheme based on diagrams expressed in terms of bare single and pair propagators with shifted chemical potential $\tilde{\mu}=\mu-U n / 2$; this is similar in spirit to the $\left[G^{(0)}\right]^{2} U$ expansion but with one extra geometrical series (bare ladder diagrams) being accounted for analytically.

To establish the parameter region where DiagMC works, we performed calculations using all four schemes. 
The results were compared to each other and to those obtained by DCA. Additional insight was also gained by doing calculations in the atomic limit [140]. We find that for bare coupling $U / t<4$ and temperature $T / t>0.1$, all schemes produce consistent results within statistical errors. At half filling, $n=1$, and $U / t \leq 6$, the $\left(G^{(0)}\right)^{2} U$ and $\left(G^{(0)}\right)^{2} \Gamma^{(0)}$ schemes still produce results consistent with those obtained by DCA and the determinant Monte Carlo method. In the dilute region (small filling factors), the $G^{2} \Gamma$ and $\left(G^{(0)}\right)^{2} \Gamma^{(0)}$ schemes can be applied for larger values of $U$. For dilute systems, our benchmarks include points $(U / t=6$, $n=0.6),(U / t=6, n=0.3)$, and $(U / t=8, n=0.3)$.

\section{EXTRAPOLATIONS AND UNCERTAINTIES}

\section{A. Extrapolations}

All of the numerical methods we have considered rely on the extrapolation of results to a thermodynamic or asymptotic limit. For DiagMC, which is formulated directly on an infinite system, the extrapolation is in diagrammatic order. All other methods are extrapolated from a finite embedded system, finite cluster, or cylinder with finite width to the infinite-system-size limit. In many cases, a considerable contribution to our errors comes from this extrapolation procedure, which differs from method to method. In some cases, determining stochastic uncertainties in extrapolation is not possible, in which case we produce estimates of uncertainties by choosing a reference system for a given technique. We then assume a normal distribution of uncertainty with respect to the reference. Specifics of the extrapolation procedure for each system (and of the associated procedure for estimating extrapolation uncertainties) are described in Sec. III. All methods have therefore defined procedures to estimate error in TL quantities as accurately as possible through the use of known reference systems. Additional uncertainties due to extrapolation, curve fitting, and truncation in excitation order are addressed on a per-technique basis. These added uncertainties are assumed to be normally distributed and defined such that they can, in principle, be made arbitrarily small by adding additional data. This section illustrates these extrapolations and presents some of the challenges encountered in performing them.

We start our discussion with ground-state properties. In Fig. 1, DMRG, FN, and AFQMC results are presented in the main panel. The system-size dependence in all three techniques is clearly visible, and the difference between the estimated thermodynamic-limit value and the largest size considered is, in some cases, outside of the error bars of the thermodynamic-limit value; in other words, extrapolation is essential for obtaining the thermodynamic-limit value. For this reason, in the results sections, we typically present both the thermodynamic-limit value and the sequence of finitesize results which led to it, so that the reader can see how large an extrapolation is required.

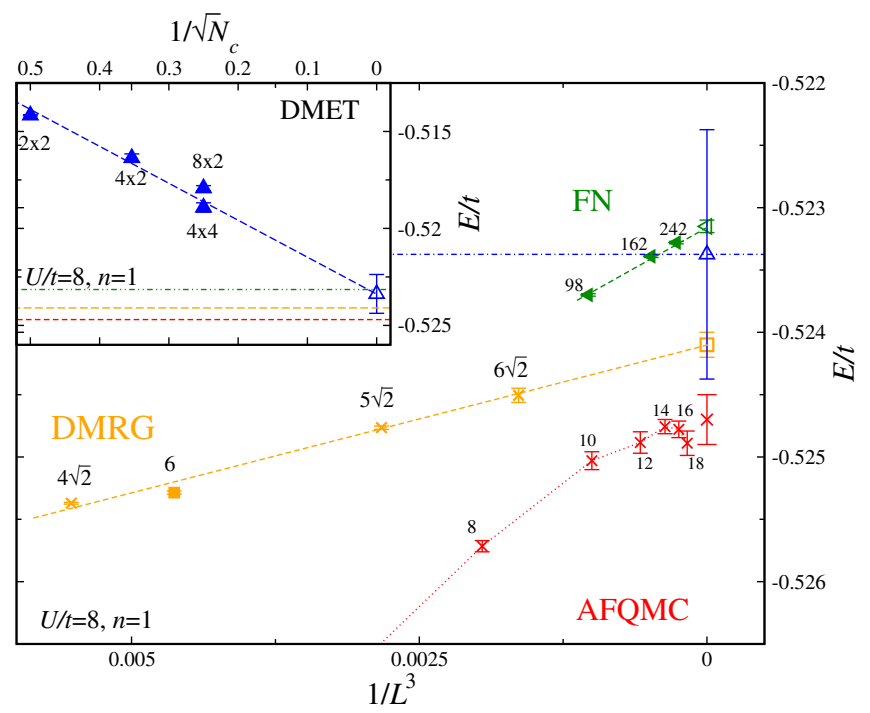

FIG. 1. Extrapolations of the ground-state energy at $U / t=8$, $n=1$. Main panel: AFQMC and FN extrapolated as a function of the inverse cube of the system's linear dimension $L$ along with DMRG extrapolated in the cube of the inverse cylinder circumference (also denoted $L$ ). DMRG data are presented both for rotated (with $\sqrt{2}$ ) and unrotated wrapping of cylinders. Inset: DMET data for clusters of size and geometry indicated, plotted against the reciprocal of the square root of the total number of sites in the cluster $N_{c}$.

For DMRG, results from both unrotated cylinders (filled symbol; other smaller- $L$ data are not shown but lie on the same scaling curve) and rotated cylinders (crosses) are consistent, both scaling as $1 / L^{3}$, although with different slopes, allowing for a clean extrapolation to the thermodynamic limit. On the other hand, the AFQMC data indicate a change in scaling for system sizes larger than $10 \times 10$ geometry under twist-averaged boundary conditions. This could mean either that unidentified complications occur in the large-system AFQMC calculations or that deviations from the $1 / L^{3}$ size dependence might occur in the DMRG data at larger cylinder sizes (i.e., that the DMRG error bar is underestimated). In this regard, it is important to note that the ground-state energy of the largest system examined in DMRG, rotated $6 \times \infty$, is within the uncertainty of the extrapolated AFQMC data.

The FN data also demonstrate a systematic dependence of the energy on system size, allowing a precise thermodynamic-limit extrapolation. The deviation of the FN results from the AFQMC and DMRG results is caused by a systematic fixed-node error, which by comparison to other methods, seems to be no more than $2 \times 10^{-3} t$.

Shown in the inset of Fig. 1 are the extrapolations in DMET which scale as $1 / \sqrt{N_{c}}$. Because of the restricted small system sizes in DMET and large $U$, the resulting uncertainty is dominated by the extrapolation. The value, also shown in the main panel, is in good agreement with DMRG and FN, and only slightly outside error bars of the AFQMC result. 


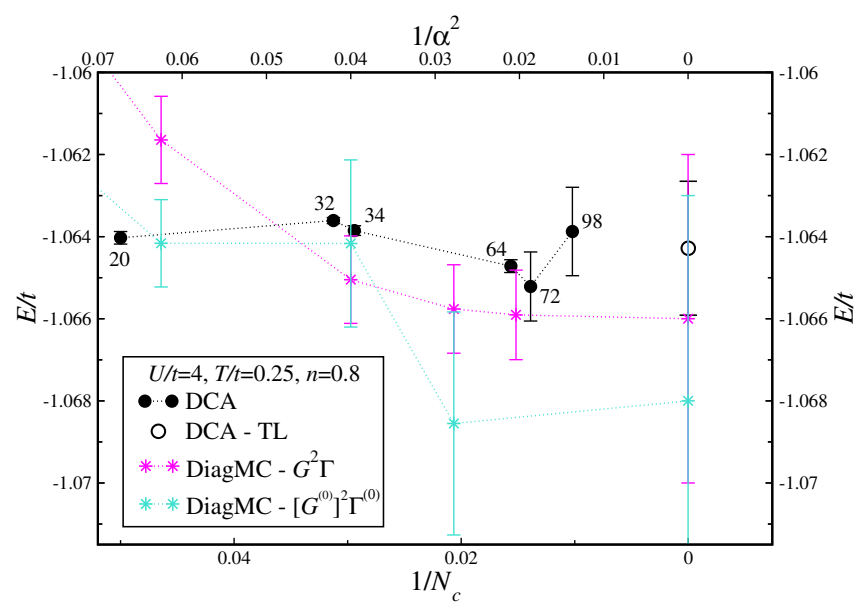

FIG. 2. Energies obtained at $U / t=4, T / t=0.25$ and $n=0.8$ from $G^{2} \Gamma$ (magenta) and $\left[G^{(0)}\right]^{2} \Gamma^{(0)}$ (turquoise) as a function of the inverse square of the diagram order parameter $\alpha$ (upper axis label) along with DCA results obtained from finite clusters plotted against the inverse of the cluster size $N_{c}$ (lower axis labels).

In Fig. 2, we show data for two DiagMC methods at $U / t=4, T / t=0.25$, and $n=0.8$, along with DCA data. DiagMC is done directly in the thermodynamic limit, and the results become successively more precise as more and more expansion orders are added to the series. The results from the two diagrammatic series we show agree, within error bars, with the $G^{2} \Gamma$ series converging more smoothly than $\left[G^{(0)}\right]^{2} \Gamma^{(0)}$. The convergence with expansion order in the regimes we present is very rapid, so the value at order $\alpha=6$ or 7 can be taken as representative of the infinite order series, with error bars estimated by statistics and by comparison to the results at the second largest order; in other words, extrapolation to $\alpha \rightarrow \infty$ is not needed.

DCA for the 2D Hubbard model approaches the thermodynamic limit $\sim 1 / N_{c}$. However, in the parameter regime considered here, the many-body physics is converged with respect to $N_{c}$ and deviations from the thermodynamic limit are dominated by single-particle shell effects. In other regimes, especially at larger $U$, extrapolation in $1 / N_{c}$ is required (see, for example, Refs. [113,114,117]).

The key result of this section is that, in many cases, extrapolation to the infinite-system-size limit is needed to obtain accurate results, with the value obtained by extrapolation significantly different from the value obtained by the largest size studied. For this reason, we typically display below both the extrapolated thermodynamic-limit results and the finite-size results that produced the extrapolation.

\section{B. Sources of uncertainty}

For clarity, we repeat the main sources of uncertainties and the meaning of the error bars shown in the graphs for each technique; further details can be found in the sections on each method. (i) AFQMC: At $n=1$, error bars include all sources of uncertainty-stochastic errors and extrapolation to TL. For $n \neq 1$, uncertainty from the constrained-path approximation is not estimated by the error bar.

(ii) FN: Error bars account for stochastic Monte Carlo errors and for extrapolation to the TL. Uncertainties due to the fixed-node approximation are not included in the error bar.

(iii) MRPHF: Results are not extrapolated to the TL, and on each finite system, an estimate of the uncertainty due to truncation in the number of Slater determinants is not included.

(iv) UCCSD: Error bars do not include an estimate of uncertainty for truncation of excitation order to doubles.

(v) DMRG: Error bars include all sources of uncertainty - the extrapolation in the number of basis states and extrapolation to TL.

(vi) DMET: Error bars include all sources of uncertainty-uncertainty due to extrapolation in the number of basis states of the impurity solver and extrapolation to TL, as well as estimates of DMET self-consistency convergence.

(vii) DCA: Error bars include all sources of uncertaintystochastic Monte Carlo uncertainties and an additional estimate of uncertainty due to extrapolation to the TL.

(viii) DF: Values are presented without error bars; the effect of neglecting nonladder and higher-order diagrams is not quantified.

(ix) DiagMC: Error bars include all sources of uncertainty - the stochastic Monte Carlo uncertainty at each expansion order and an estimate of the uncertainty in convergence of expansion order.

\section{RESULTS AT INTERMEDIATE TO STRONG INTERACTION STRENGTHS}

We begin our discussion of results with an analysis of an intermediate to strongly coupled case, namely, $U / t=8$. Throughout all the figures, we use common legends, distinguishing techniques by symbol and color. We present both results for the thermodynamic-limit and the finitesystem-size data from which the thermodynamic-limitresults were obtained. This information is useful in assessing both the importance of the extrapolation and other aspects of the performance of the method. We use open symbols to denote values in the thermodynamic limit and filled symbols for finite-size values from which the extrapolations are obtained.

\section{A. Half-filled, particle-hole symmetric case $\left(U / t=8, n=1, t^{\prime} / t=0\right)$}

We begin our discussion with an analysis of the energy per site for a widely accessible parameter choice, half filling, showing in Fig. 3 the temperature dependence of the energy and in Fig. 4 an expanded view of the $T=0$ energy. 


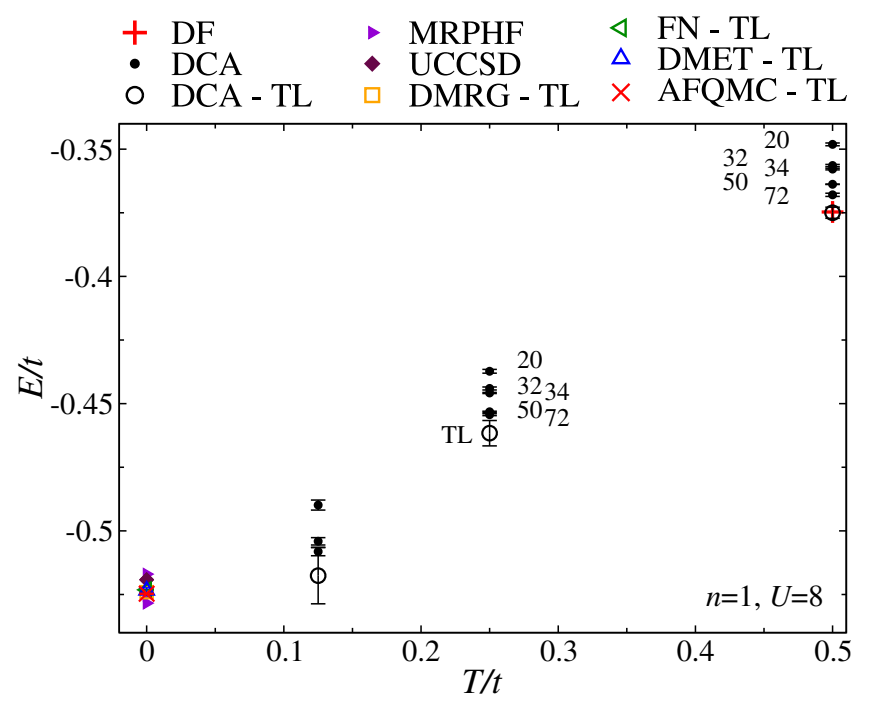

FIG. 3. Temperature dependence of the energy for $n=1$ for $U / t=8$ obtained by DCA (black circles) and DF (red cross) and compared to zero-temperature results compiled from various techniques. Solid symbols represent finite systems; open symbols represent extrapolations to the TL.

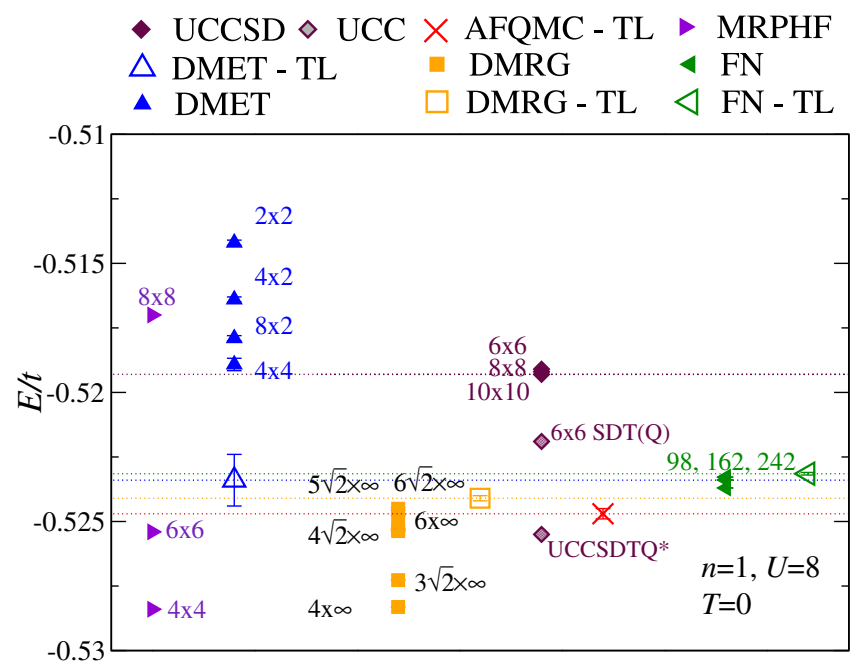

FIG. 4. Thermodynamic-limit ground-state energy for $n=1$ for $U / t=8$ as obtained by various algorithms (open symbols). Also shown are the finite-size systems (filled symbols with adjacent labels) from which the TL ground-state energy was obtained. Data are from AFQMC (red crosses), DMET (blue triangles), UCCSD (maroon diamonds), MRPHF (purple triangles), DMRG (orange squares), and FN (green triangles). Horizontal thin dotted lines show the best estimates for the ground-state energy.

First, we discuss the DCA results in Fig. 3; in this particle-hole symmetric parameter regime, the impurity solvers have no sign problem, and have cubic complexity, meaning that reliable results can be obtained on relatively large clusters and that the Monte Carlo errors (here, on the order of $10^{-3}$ ) can be systematically reduced with additional computation. Thermodynamic-limit data are obtained from the $1 / N_{c}$ extrapolation. Computational scaling towards low temperatures results in an increase of uncertainty for fixed computational time, and this is reflected in the uncertainty in the extrapolated values. At $T / t=0.5$, our results agree within error bars with hightemperature series and lattice Monte Carlo data (see Ref. [114]).

The results of a DF calculation are shown at $T / t=0.5$ (lower $T$ data are not available). The DF technique neglects vertex functions of higher order than two-particle vertices. Furthermore, at the two-particle level, we sum only a ladder series in the spin and charge channels. Despite these approximations, we see that the DF technique provides an energy which falls on top of that of the extrapolated thermodynamic-limit DCA result.

Results from a variety of algorithms are available at zero temperature. Figure 4 presents an expanded view of the $T=$ 0 results, with the energy on the vertical axis and data for each method offset in the $x$ axis. Note that in some cases, the thermodynamic-limit results are further offset for clarity.

We start our discussion of zero- $T$ results with a Monte Carlo technique, AFQMC, which is extrapolated to the thermodynamic limit. In this case, finite-size results are averaged over twisted-boundary conditions, which allows a smooth and rapid convergence to the thermodynamic limit. These results, obtained at half filling from Monte Carlo, are unbiased and therefore expected to be exact within a quoted uncertainty of $\pm 0.0002 t$.

DMRG results on cylinders of infinite length but finite width of $3,4,5$, and 6 for $45^{\circ}$ rotated systems and width of 4 and 6 for nonrotated systems are shown. All the finite-size data are after phase averaging, showing only very weak finite-size effects so that an extrapolation to the thermodynamic limit is feasible. In this case, the estimation of uncertainty (as discussed in Sec. III F) contains the uncertainty of each extrapolation and the difference between the two orientations (rotated and nonrotated), both of which are on the order of $10^{-4} t$. The resulting energy is close to, but slightly outside of, the AFQMC results. This extrapolation issue was discussed in more detail in Sec. IVA.

For DMET, we show results obtained for finite clusters of size $2 \times 2,4 \times 2,8 \times 2$, and $4 \times 4$. The thermodynamic limit is obtained by extrapolating the $2 \times 2,4 \times 2$, and $4 \times 4$ clusters in $1 / \sqrt{N_{c}}$. Errors from the solution of the finite impurity are on the order of $10^{-4} t$. DMET cluster-size convergence is slower at large $U$; thus, $U / t=8$ corresponds to the largest half-filling DMET error bar discussed here. The total thermodynamic-limit uncertainty is estimated to be $0.001 t$ and comes entirely from the thermodynamic-limit extrapolation. The lower end of the DMET error bar lies at the average of the DMRG and AFQMC estimates.

For the FN technique, a diffusion Monte Carlo calculation based on the nodal structure of a trial wave function 
obtained with variational Monte Carlo, we show finite-size results for a sequence of 45-degree rotated clusters with size 98,162 , and 242, which have the property of being closed shells at $U=0$. The results show only weak size dependence, so the thermodynamic-limit value shown is close to the finite-size results. However, the results are systematically above the values obtained by AFQMC and DMRG, while they are consistent with DMET. This is a consequence of the fixed-node approximation, which, in this particular case, resulted in a fixed-node error of about $0.0015 t$.

The results of UCCSD are shown for systems of size $6 \times 6,8 \times 8$, and $10 \times 10$, and exhibit weak finite-size effects at this $U$ value. We see that the result is accurate to roughly the $1 \%$ level. The deviation is caused by correlations that are not captured by singles and doubles. Higher-order excitations (triples, quadruples, etc.) will eventually recover the remaining energy. To support this claim, we show a single case in Fig. 4 labeled as UCCSDT(Q), which includes all triples and a subset of quadruples. In this higherorder approximation, the deviation from other techniques is reduced by a factor of 2 . The higher-order corrections are more important for these coupled cluster calculations than extrapolations in cluster geometry size; however, the improvement with increased excitation order converges slowly. Also shown are approximate results including quadruples from small system sizes $(4 \times 4)$, which we label UCCSDTQ*. While not exact, this approximation scheme produces results that deviate from AFQMC by only $0.15 \%$.

MRPHF calculations have been performed for several finite systems $(4 \times 4,6 \times 6$, and $8 \times 8)$. As summarized in the methods description, reaching a constant level of accuracy would require a successively larger MR expansion. Results for larger systems are therefore solved less precisely; in particular, the energy of the $8 \times 8$ lattice in Fig. 4 is too high. More sophisticated implementations and additional optimizations may make it possible to reach the accuracy needed to perform extrapolations to the thermodynamic limit.

We now discuss the results for the double occupancy in Fig. 5 at $U / t=8$ and $n=1$. Open symbols denote results in the thermodynamic limit, filled symbols results on finite systems. The finite- $T$ DCA results show that the double occupancy contribution rises as the temperature is lowered. The finite- $T$ results are consistent with the $T=0$ values obtained by AFQMC, DMRG, DMET, FN, and MRPHF. At $T / t=0.5$, the double occupancy obtained from the DF technique is also shown. Unlike the total energy, the DF double occupancy shows deviations from the DCA result, suggesting a cancellation of errors in the kinetic and potential energy terms. As for all other points we have examined, the DF method produces results that lie between single-site DMFT values (not shown) and the extrapolated DCA results.

The inset shows the various $T=0$ values. Within error bars, there is agreement between AFQMC, DMET, and

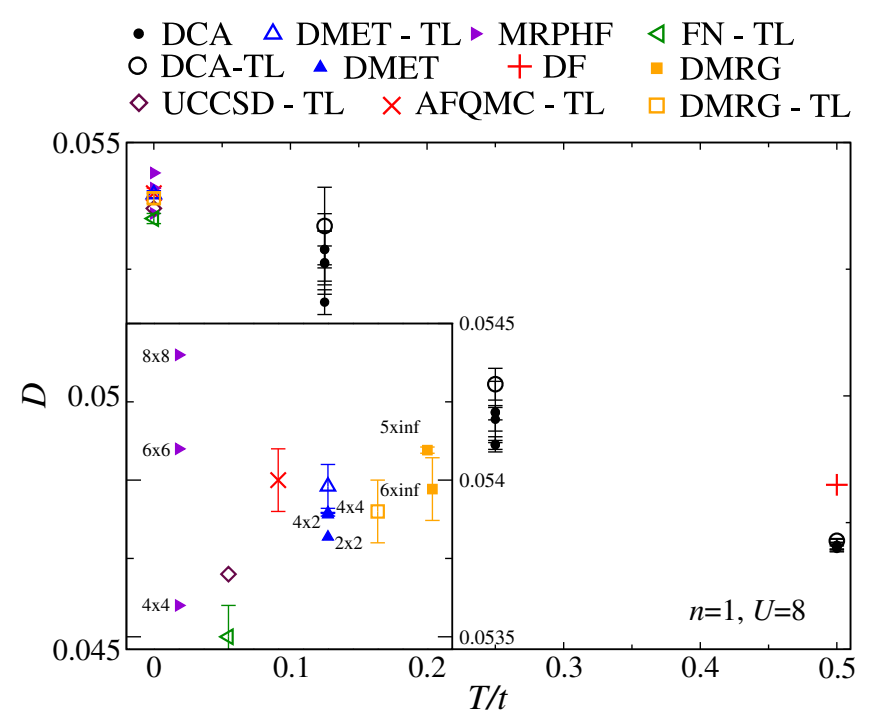

FIG. 5. Double occupancy data for $U / t=8$ and $n=1$. Main panel: Temperature dependence of double occupancy, obtained from DCA (finite $T$, black circles) and DF (finite $T$, red plus sign), and the $T=0$ techniques AFQMC (red crosses), DMET (blue triangles), UCCSD (maroon diamonds), MRPHF (purple triangles), DMRG (orange squares), and FN (green triangles). Solid symbols represent finite systems; open symbols represent extrapolations to the thermodynamic limit. Inset: Data at $T=0$ reproduced with an arbitrary $x$-axis offset, from MRPHF, UCCSD, FN, DMET, DMRG, and AFQMC.

DMRG results for the double occupancy. DMET obtains a value (after thermodynamic-limit extrapolation) comparable to AFQMC and, overall, shows a weaker systemsize dependence than for the energy.

UCCSD and FN produce a double occupancy that is underestimated as compared to AFQMC and DMET. Finite-size effects of FN are on the order of 0.0001 . Finally, for MRPHF, we quote two values for $4 \times 4$ and $6 \times 6$ systems which show a system-size dependence on the order of 0.001 . This makes a thermodynamic-limit extrapolation impractical.

\section{B. Doped strongly correlated regime $\left(U / t=8, n=0.875, t^{\prime} / t=0\right)$}

The half-filled particle-hole symmetric case of Sec. VA is, in many ways, ideally suited for numerical algorithms: A large charge gap allows methods like the DMRG to quickly converge, and particle-hole symmetry makes Monte Carlo simulations without a sign problem possible. We now turn to a case that is particularly difficult to simulate, where we expect results to be substantially less accurate than for the half-filled case. This parameter regime shows metallic behavior, strong particle-hole asymmetry, and interesting inhomogeneous phases in the ground state. In Fig. 6, we plot the total energy per site and in Fig. 7 the double occupancy per site at $U / t=8$ and $n=0.875$.

The main panel presents data as a function of temperature. The DCA results remain consistent with $T=0$, but 


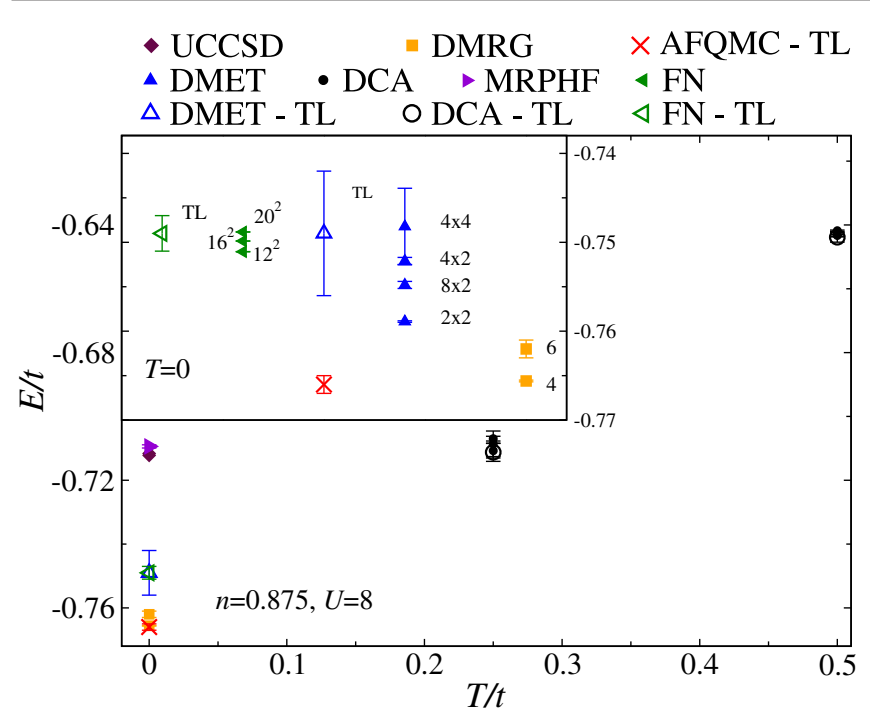

FIG. 6. Data for $n=0.875$ for $U / t=8$. Main panel: Temperature dependence of $E / t$ compiled from various techniques. Solid symbols represent finite systems; open symbols represent extrapolations to the thermodynamic limit. Finite- $T$ results are shown for DCA (black circles), and zero- $T$ data from AFQMC (red crosses), DMET (blue triangles), UCCSD (maroon diamonds), DMRG (orange squares), and FN (green triangles). Top left inset: Zoom-in of the zero- $T$ data from DMET, AFQMC, FN, and DMRG including finite-system-size data (as labeled).

results are not available at the lowest benchmark temperature, $T / t=0.125$, because of a large sign problem in the Monte Carlo impurity solver. The finite-size effects, at the system sizes accessible in DCA, are smaller than in the $n=1$ case.

The inset to Fig. 6 presents data at $T=0$ with an arbitrary $x$-axis offset added for clarity. The AFQMC simulation, using a (nonvariational) constrained-path approximation in the absence of particle-hole symmetry, yields a result for the total energy that is lower than the one obtained from DMET, FN, and DMRG. The total energy difference is about $1 \%$ when compared to finite-size DMRG, and about $1.4 \%$ (2.1\%) when compared to the DMET $8 \times 2$ (thermodynamic-limit) cluster, respectively, and about $2.1 \%$ in comparison to FN.

DMRG shows the results for cylinders of infinite length and finite widths of 4 and 6 lattice sites after using phase averaging. The energy is higher for the wider cylinder, and for the width-6 cylinder, the energy is above the energy from AFQMC. Given that the extrapolation is performed with only two widths, we consider the extrapolated DMRG value to be unreliable in this case and omit it entirely.

DMET shows a large-system-size dependence and a dependence of the thermodynamic-limit value on the cluster sequence chosen for the extrapolation. We show an extrapolation based on $2 \times 2,4 \times 2$, and $8 \times 2$ clusters. The use of the $8 \times 2$ cluster allows inhomogeneous order to develop, giving an extrapolated value of $E / t=-0.749$ (7). The extrapolation using the $4 \times 4$ rather than $8 \times 2$ cluster,

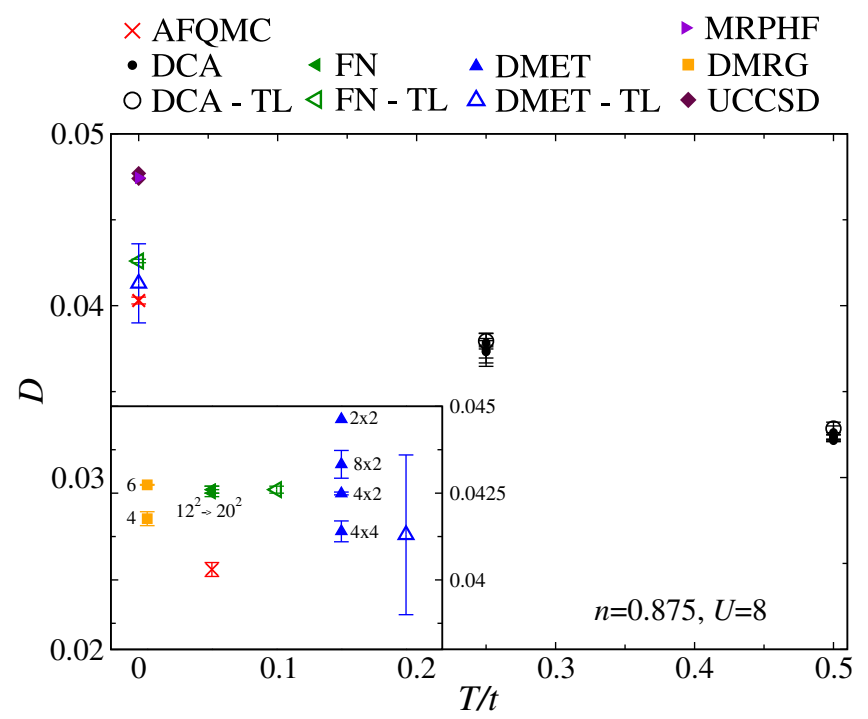

FIG. 7. Data for $n=0.875$ for $U / t=8$. Main panel: Temperature dependence of double occupancy $D$, compiled from various techniques. Solid symbols represent finite systems; open symbols represent extrapolations to the thermodynamic limit. Finite- $T$ results are shown for DCA (black circles), and zero- $T$ data from DMET (blue triangles), MRPHF (purple triangles), UCCSD (maroon diamonds), DMRG (orange squares), and FN (green triangles). Inset: Zoom-in of the zero- $T$ data from FN, AFQMC, DMET, and DMRG.

which does not allow for inhomogeneous order, yields a value of $E / t=-0.737(5)$. Since the energy changes nonmonotonically-the $8 \times 2$ energy lies above the $2 \times 2$ energy but below the $4 \times 2$ energy - the uncertainty in the thermodynamic-limit extrapolation is very large and does not provide any more information than the results obtained from the largest clusters.

The FN method shows a clear finite-system-size dependence. The infinite system value is estimated from the $16 \times$ 16 and $20 \times 20$ values, and finite-size errors are on the order of $0.001 t$, much larger than the stochastic errors of $0.00001 t$. Here, the FN results are consistent with DMET extrapolation, which omits the $4 \times 4$ cluster, and these are considerably higher than AFQMC. This is suggestive of a fixed-node error of $\approx 0.015 t$, indicating that a uniform variational wave function may not fully account for the nature of the ground state. Indeed, the variational Monte Carlo (VMC) error is of the order of $0.022 t$, much larger than the one obtained at half filling, which was $\approx 0.004 t$. In both cases, the FN projection improves the $\mathrm{VMC}$ results by the same order of magnitude.

UCCSD and MRPHF results are much higher in energy $[E / t=-0.7094(5)$ for MRPHF $(16 \times 4$ system $)$ and $E / t=-0.7122$ for UCCSD, barely visible on the main panel], an indication that correlated metallic states are difficult to capture with these methods. Although not shown, data are available for $\operatorname{UCCSD}(\mathrm{T})$ (perturbative inclusion of triples) in Ref. [46], which improves upon 
the value from UCCSD and gives $E / t=-0.7272$ $(-0.7281)$ for a $16 \times 4(16 \times 8)$ cluster. Full inclusion of triples (UCCSDT) lowers the $16 \times 4$ estimate to $E / t=-0.7427$. The MRPHF results indicate the need for a much larger MR expansion than that afforded in this work.

In this parameter regime, ordered "stripe" phases might exist. However, the precise form of these stripes is strongly influenced by choice of finite-size systems (e.g., width and orientation of the cylinder in DMRG and shape of the cluster in DMET) that are used for the thermodynamic extrapolation and the approximations used to solve that finite system. The finite-temperature algorithms have not reached the onset of inhomogeneous states at the lowest temperature accessible. The precise nature of the inhomogeneities in the ground state in this parameter regime is still open.

Finally, we briefly mention the results for double occupancy in Fig. 7 for $U / t=8$ and $n=0.875$. As was the case for the energies, the finite- $T$ results smoothly connect to the zero- $T$ values. MRPHF and UCCSD overestimate the double occupancy by close to $15 \%$. The remaining ground-state methods (DMRG, AFQMC, DMET, and FN) present consistent values in the range from 0.04 to 0.043 . Both $\mathrm{FN}$ and AFQMC values contain additional (fixed-node and constrained-path) errors that are not estimated by the error bar.

\section{Half-filled, non-particle-hole symmetric case $\left(U / t=8, n=1, t^{\prime} / t=-0.2\right)$}

We now turn our attention to a case of half filling without particle-hole symmetry, by adding a second nearestneighbor hopping $t^{\prime}$. An overview of the energies from several algorithms for $U / t=8, n=1$, and $t^{\prime}=-0.2$ is shown in Fig. 8.

The main panel shows the temperature dependence of the data. The DCA results available at finite $T$ show almost no sign problem for $T / t=0.5$ and $T / t=0.25$ but are hampered by a severe sign problem at $T / t=0.125$. The results are consistent within error bars with the zerotemperature results.

As at $U / t=8, n=0.875, t^{\prime}=0$, the AFQMC is approximate because of a constrained-path approximation due to the lack of particle-hole symmetry. Despite this, the results are in agreement with both the DMET and DMRG results.

The DMET results are obtained on clusters of size $2 \times 2$, $2 \times 4$, and $4 \times 4$. Errors of the individual finite-size systems are substantially smaller than the system-size dependence. The DMET thermodynamic limit is consistent with the thermodynamic estimates obtained from DMRG (from cylinders of width 4 and 6) and from AFQMC. This is even more evident in the bottom right inset, which displays the thermodynamic-limit estimates on a smaller scale.

FN results are higher in energy than AFQMC and DMET (well within 2 joint standard deviations) and are higher than DMRG by $0.0013 t$. As seen in previous plots, the

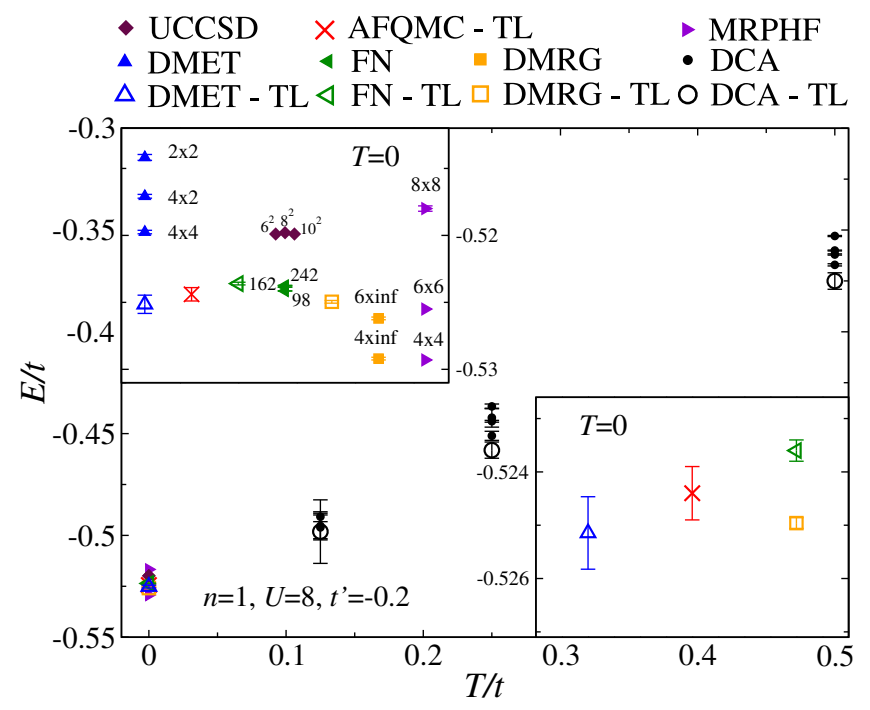

FIG. 8. Data for $n=1$ for $U / t=8$ with $t^{\prime} / t=-0.2$. Main panel: Temperature dependence of $E / t$ compiled from various techniques. Solid symbols represent finite systems; open symbols represent extrapolations to the thermodynamic limit. Finite- $T$ results are shown for DCA (black circles), and zero- $T$ data from AFQMC (red crosses), DMET (blue triangles), UCCSD (maroon diamonds), MRPHF (purple triangles), DMRG (orange squares), and FN (green triangles). Top left inset: Zoom-in of the zero- $T$ data from MRPHF, UCCSD, DMET, FN, DMRG, and AFQMC, including finite-system-size data (as labeled) for MRPHF, FN, DMET, and DMRG. Bottom right panel: Enlarged region of the top left inset showing DMET, DMRG, FN, and AFQMC data at $T=0$, including error bars, extrapolated to the infinite system size.

finite-system-size dependence of the fixed-node results is small on this scale.

UCCSD results show only small finite-size effects and an overall energy $\approx 1 \%$ higher than other techniques. The MRPHF results obtained on finite systems show an energy that rises rapidly as the system size is increased. As in the case of $t^{\prime}=0$, a systematic extrapolation to the thermodynamic limit is not possible, and we only present results on finite systems.

\section{RESULTS IN THE INTERMEDIATE COUPLING REGIME}

In this section, we repeat the previous discussion for an interaction strength of half the size, $U / t=4$. As before, we start our discussion at half filling. We then discuss a correlated metallic case with $20 \%$ doping.

\section{A. Half-filled, particle-hole symmetric case $\left(U / t=4, n=1, t^{\prime} / t=0\right)$}

In Fig. 9, we report the energy as a function of temperature. At finite $T$ and $U / t=4$, both DCA and the diagrammatic Monte Carlo method for the $\left[G^{(0)}\right]^{2} U$ series provide results in the thermodynamic limit. DCA results in 
the thermodynamic limit are extrapolated from finite clusters; DiagMC results are extrapolated in the expansion order. The results are consistent within the error bars of the respective methods. The large error bars of the extrapolation in DiagMC- $\left[G^{(0)}\right]^{2} U$ mainly come from a conservative estimate of the diagram-order extrapolation error. DCA shows surprisingly large finite-size effects which persist above $N_{c}=72$, unlike at $U / t=8$. While each individual $N_{c}$ result has uncertainties in the energy on the order of $10^{-4} t$, the spread in values results in a large uncertainty when extrapolated to the thermodynamic limit.

We now move to the zero-temperature methods, which are shown in the inset of Fig. 9. AFQMC provides numerically exact ground-state energies for this system. The value quoted is $E / t=-0.8603(2)$, which is in agreement with the DMET value of $E / t=-0.8604$ (3) and the DMRG value of $E / t=-0.8605(5)$. DMET values are obtained from an extrapolation of $2 \times 2,2 \times 4$, and $4 \times 4$ clusters. DMRG values are obtained from an extrapolation of widths 3,4 , and 5 for 45 -degree rotated cylinders and of widths 4 and 6 for nonrotated cylinders.

The results obtained by AFQMC, DMRG, and DMET are in excellent agreement with recent calculations obtained from linearized auxiliary-field Monte Carlo (LAQMC) available in the literature [141], which gives $E / t=-0.85996(5)$. FN results are higher in energy

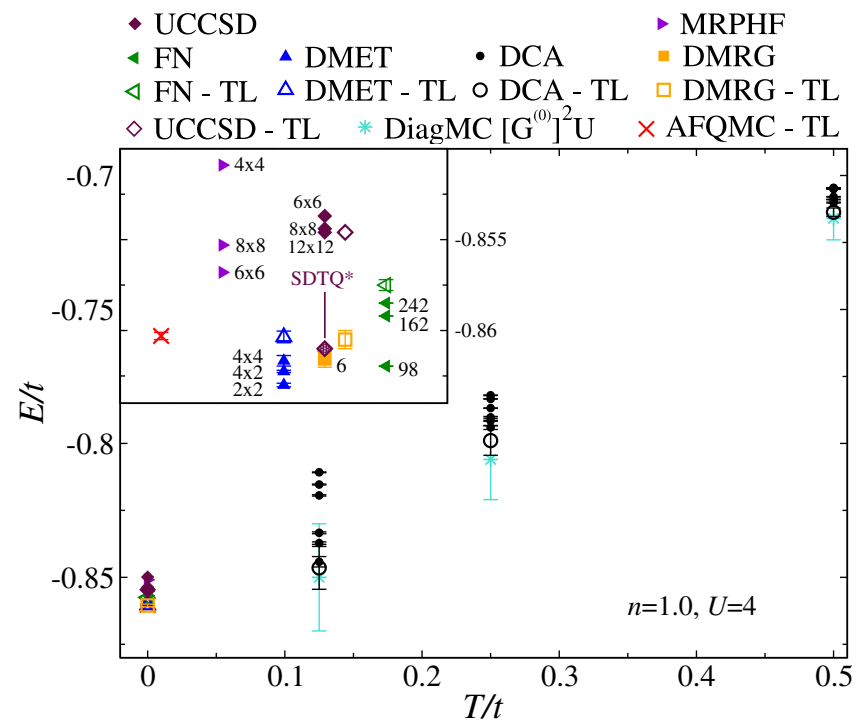

FIG. 9. Data for $n=1$ for $U / t=4$. Main panel: Temperature dependence of $E / t$ compiled from various techniques. Solid symbols represent finite systems; open symbols represent extrapolations to the thermodynamic limit. Finite- $T$ results are shown for DCA (black circles) and DiagMC (turquoise stars), and zero$T$ data from AFQMC (red crosses), DMET (blue triangles), UCCSD (maroon diamonds), MRPHF (purple triangles), DMRG (orange squares), and FN (green triangles). Top left inset: Zoomin of the zero- $T$ data from MRPHF, DMET, FN, DMRG, and AFQMC, including finite-system-size data (as labeled) for MRPHF, FN, DMRG, and DMET.

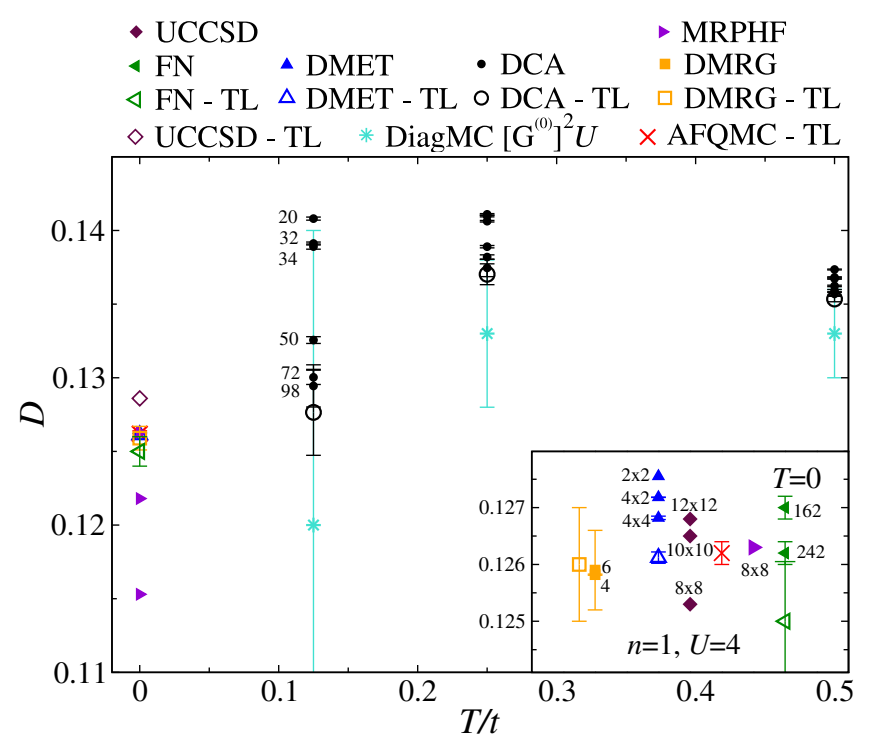

FIG. 10. Data for $n=1$ for $U / t=4$. Main panel: Temperature dependence of double occupancy $D$, compiled from various techniques. Solid symbols represent finite systems; open symbols represent extrapolations to the thermodynamic limit. Finite- $T$ results are shown for DCA and DiagMC (turquoise stars), and zero- $T$ data from DMET (blue triangles), UCCSD (maroon diamonds), MRPHF (purple triangles), DMRG (orange squares), and FN (green triangles). Inset: Zoom-in of the zero- $T$ data from DMRG, FN, UCCSD (only finite-system data), DMET, and AFQMC.

$[E / t=-0.8575(3)]$, and unlike in previous cases for stronger interaction, a clear dependence on the finite system studied is visible. The FN projection technique leads to an energy gain of $\approx 0.002 t$ with respect to the $\mathrm{VMC}$ result of $E / t=-0.8558(5)$. This number can be compared with a previous estimation of the thermodynamic limit in VMC obtained with a slightly less accurate variational state (see Ref. [78]).

The UCCSD thermodynamic limit overestimates the energy by about $0.7 \%$. MRPHF values, shown as purple triangles in the main panel and inset, show large finite-size effects and are higher than the values obtained with other methods. We see that as the system size is increased, the energy increases rapidly.

In Fig. 10, we report the double occupancy vs $T$. At finite $T$, the DCA results show a clear rise in $D$ as $T$ decreases from $0.5 t \rightarrow 0.25 t$. However, this trend reverses as $T$ decreases further. A similar behavior is also obtained by using DiagMC, demonstrating that this is a genuine effect present in the Hubbard model.

These trends are consistent with the $T=0$ data, which lie below all of the DCA data points at finite $T$. For clarity of presentation, we again display this data in the inset and add an arbitrary $x$-axis offset. We see that finite-size effects in DMET are very small and that the extrapolation of DMET agrees perfectly with AFQMC. Finite-size FN results produce values comparable to DMRG, DMET, 


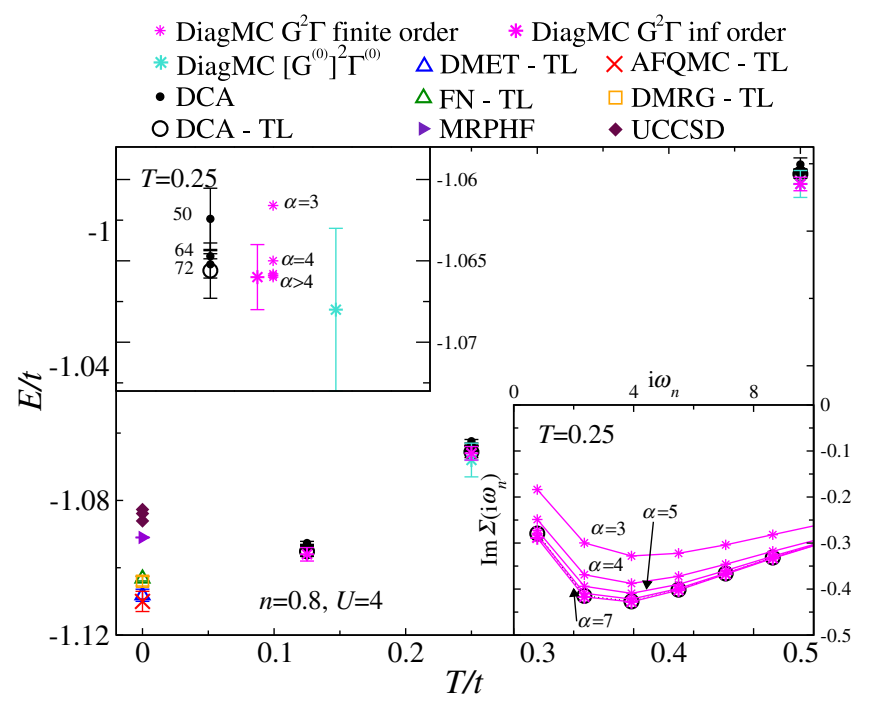

FIG. 11. Data for $n=0.8$ for $U / t=4$. Main panel: Temperature dependence of $E / t$ compiled from various techniques. Solid symbols represent finite systems; open symbols represent extrapolations to the thermodynamic limit. Finite- $T$ results are shown for DCA (black circles) and DiagMC (pink and turquoise asterisks), and zero- $T$ data from AFQMC (red crosses), DMRG (orange squares), FN (green triangles), and DMET (blue triangles). Top left inset: Zoom-in of the $T / t=0.25$ data from DCA and two types of DiagMC for different orders $\alpha=3,4, \ldots$. Bottom right inset: Plot of the imaginary part of the local selfenergy $\operatorname{Im} \Sigma\left(i \omega_{n}\right)$ from DiagMC for different expansion orders $\alpha$ and from DCA (black circles covered by magenta stars).

and AFQMC. However, extrapolation in FN results in an underestimate of the double occupancy, although within uncertainties. In the DMRG simulations, phase averaging has greatly reduced finite-size effects, and the DMRG error bars are determined by the truncation errors. Within those error bars, DMRG results are consistent with AFQMC and DMET.

\section{B. Doped case $\left(U / t=4, n=0.8, t^{\prime} / t=0\right)$}

Away from half filling (with $t^{\prime}=0$ ), we can perform further comparisons at finite $T$ between DCA and DiagMC at $U / t=4$. We begin the discussion with the inset of Fig. 11, which shows the convergence of the imaginary part of the local Matsubara self-energy of the $G^{2} \Gamma$ and $G^{2} W$ DiagMC series as a function of evaluation order. The values are compared to DCA results. We see that the first six orders of the series are precise enough to get good agreement of the Matsubara selfenergy in the thermodynamic limit, and convergence is rapid. While deviations are visible in the energy, these are attributed to differences of the chemical potential, i.e., the real part of the self-energy.

The top left inset of Fig. 11 shows the convergence of the energy in DCA and two different series of DiagMC, $\left[G^{(0)}\right]^{2} U$ and $G^{2} \Gamma$, for increasing order of the diagrammatic

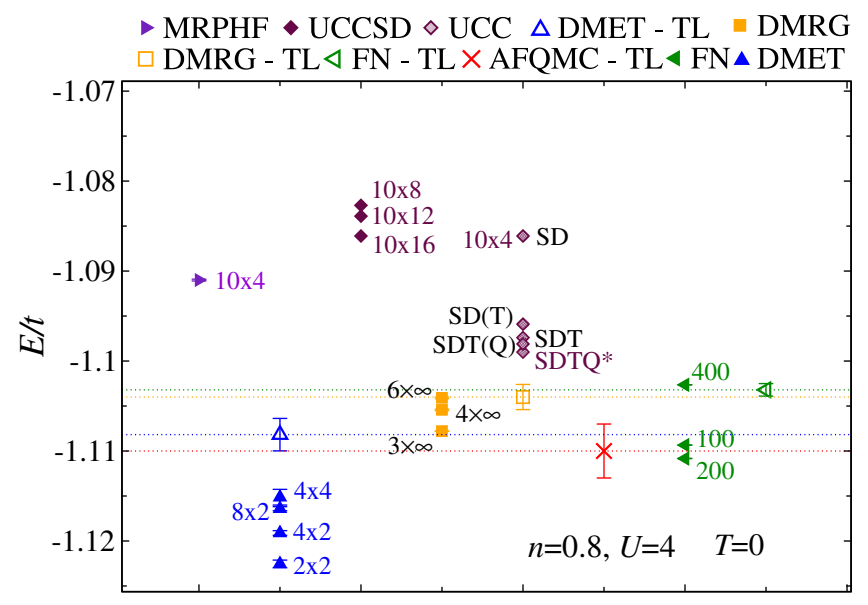

FIG. 12. Thermodynamic-limit ground-state energy for $n=0.8$ for $U / t=4$ as obtained by various algorithms (open symbols). Also shown are the finite-size systems (filled symbols with adjacent labels) from which the thermodynamic-limit groundstate energy was obtained. Data are from AFQMC (red crosses), DMET (blue triangles), UCCSD (maroon diamonds), UCC on a $10 \times 4$ lattice (shaded diamonds), MRPHF (purple triangles), DMRG (orange squares), and FN (green triangles). Horizontal thin dotted lines show the best estimates for the ground-state energy.

resummations, $\alpha$. The values obtained from the three techniques are within error bars.

The $T>0$ values smoothly connect to $T=0$ (although a precise comparison is not possible because we lack a quantitative functional form to extrapolate the $T>0$ values to $T=0$ ), which we display separately in Fig. 12, where data from DMET, AFQMC (constrained path), UCCSD, MRPHF, FN, and DMRG are shown. In this case, MRPHF and UCCSD are systematically higher in energy from the other techniques. In the case of UCCSD, we see larger finite-size effects than at $U / t=8$. Inclusion of higher orders of excitation [perturbative triples $(\mathrm{T})$, triples $\mathrm{T}$, and perturbative quadruples (Q)] suggests that the dominant error is associated with the truncation of the excitation order and not with finite size effects. The FN result is in agreement with the value from DMRG. At slightly lower energy, AFQMC (constrained path) and DMET are in close agreement. Overall, the spread in energies is similar to that shown away from half filling at $U / t=8$ (see Fig. 6) but smaller in magnitude, perhaps due to better convergence for weak coupling in some techniques.

\section{RESULTS IN THE WEAK COUPLING REGIME}

In the weak coupling limit, we restrict the presentation of data to the half-filled case since the correlated metallic phase is not qualitatively distinct from $U / t=4$. Data sets for doped, weakly correlated systems are available in Ref. [46]. 


\section{A. Half-filled, particle-hole symmetric case

$$
\left(U / t=2, n=1, t^{\prime} / t=0\right)
$$

In Figs. 13 and 14, we present results for $U / t=2$ and $n=1$, the half-filled weak coupling regime. This regime is particularly easy for methods based on a weak coupling expansion around a noninteracting system, and many of the algorithms show uncertainties that are much smaller than in the intermediate or strong interaction limit.

At nonzero $T$, the data from two types of DiagMC and from DCA in the thermodynamic limit agree within uncertainty. The values smoothly connect to the $T=0$ values, except for MRPHF energies, that are higher than the ground-state energies obtained by the other methods and higher than the energies obtained for the lowest-temperature point obtained from both finite- $T$ methods.

At $T / t=0.5$, we show a result from DF. As was the case in the strong coupling regime, the DF procedure produces an energy estimate consistent with DCA results. In this case, with only weak finite-size dependence, the underlying DMFT approximation differs from DCA by only $0.4 \%$. The DF value improves on the DMFT and differs from the extrapolated DCA results by only $0.07 \%$.

In the lower right inset of Fig. 13, we present $T=0$ extrapolations. As in the case of larger interactions, DMET and $\mathrm{AFQMC}$ (which is numerically exact in this situation)

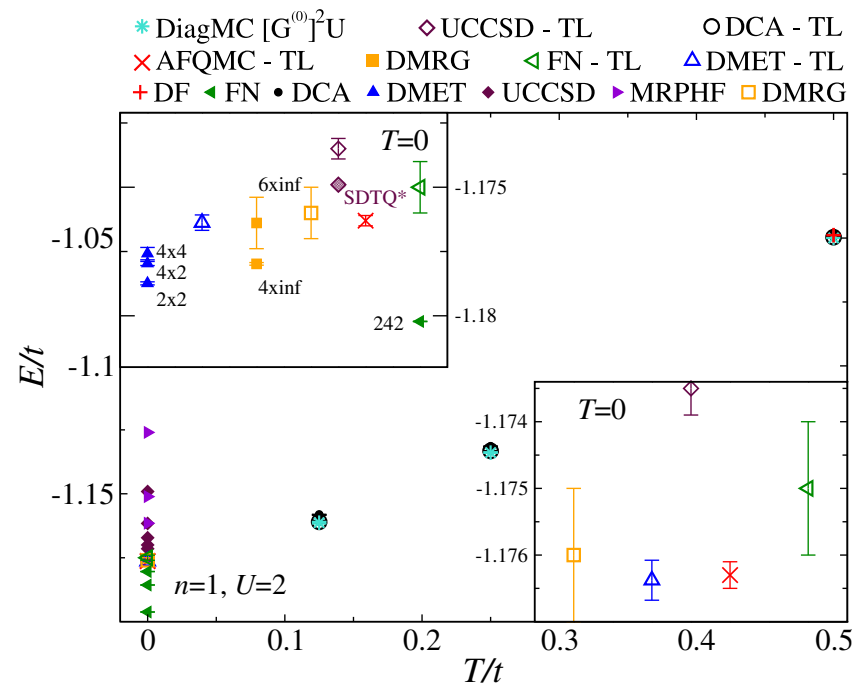

FIG. 13. Data for $n=1$ for $U / t=2$. Main panel: Temperature dependence of $E / t$ compiled from various techniques. Solid symbols represent finite systems; open symbols represent extrapolations to the thermodynamic limit. Finite- $T$ results are shown for DCA (black circles) and DiagMC (blue asterisks), and zero- $T$ data from AFQMC (red crosses), DMET (blue triangles), UCCSD (maroon diamonds), MRPHF (purple triangles), DMRG (orange squares), and FN (green triangles). Top left inset: Zoomin of the zero- $T$ data from UCCSD, DMET, FN, DMRG, AFQMC, and DMET. Bottom right panel: Enlarged region of the top left inset showing thermodynamic-limit data for DMRG, DMET, UCCSD, FN, and AFQMC at $T=0$, including error bars, extrapolated to the infinite system size. agree precisely, while FN is slightly higher in energy but compatible within two error bars. In the upper left inset, we explore the finite-size effects of the methods. In the case of DMET, these finite-size effects are small and can be extrapolated with small error bars. FN shows much larger finite-size effects, approaching the thermodynamic-limit energy from below (only the largest system size is visible on the scale of the plot). DMRG results with phase averaging are precise even at $U / t=2$, though much larger uncertainties than at $U / t=8$ are present.

The results of MRPHF, outside of the scale shown by the inset, show a gradual decrease of the energy with increasing system size: $4 \times 4$ yields $E / t=-1.1260,6 \times 6$ yields $E / t=-1.1515, \quad$ and $8 \times 8$ yields $E / t=-1.1629$. UCCSD results, shown here for cluster sizes of $12 \times 12$, $10 \times 10,8 \times 8$, and $6 \times 6$, show large finite-size effects. With the aid of an extrapolation, the value in the thermodynamic limit is estimated to be higher than other techniques. The deviation of the thermodynamic-limit value from AFQMC and DMET is on the order of $2 \times 10^{-3} t$, suggesting that excitations beyond the single and double levels are important even in this relatively weak coupling regime.

Finally, Fig. 14 shows the double occupancy for these parameters. We see an increase in finite-size effects in DCA as we progress to lower temperatures. Reasonable agreement with DiagMC is achieved in the double occupancy. At temperatures lower than our lowest temperature, the double occupancy will need to dip, as was the case at

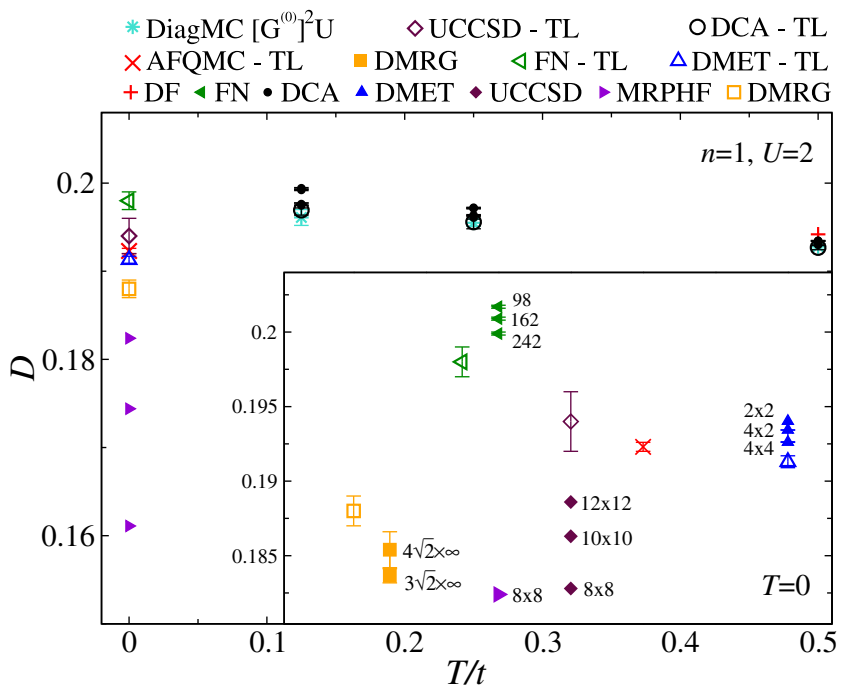

FIG. 14. Data for $n=1$ for $U / t=2$. Main panel: Temperature dependence of double occupancy $D$, compiled from various techniques. Solid symbols represent finite systems; open symbols represent extrapolations to the thermodynamic limit. Finite- $T$ results are shown for DCA (black circles) and DiagMC (turquoise asterisks), and zero- $T$ data from DMET (blue triangles), UCCSD (maroon diamonds), MRPHF (purple triangles), DMRG (orange squares), and FN (green triangles). Inset: Zoom-in of the zero- $T$ data from FN, UCCSD, MRPHF, DRMG, DMET, and AFQMC. 
$U / t=4$, in order for the finite- $T$ data to be consistent with $T=0$. Similar to the strong coupling case, DF provides only a slight shift to the double occupancy, a minimal improvement over DMFT alone.

The ground-state double occupancies are very precisely determined by AFQMC and DMET, which are in agreement. The FN value is somewhat overestimated. Results from DMRG fall below AFQMC and DMET, and the error bar underestimates the uncertainty. The larger error appears to be consistent with the difficulty in treating the small $U$ limit in the DMRG calculations. The results from MRPHF show an improvement in $D$ as the system size is increased, consistent with the behavior for the energy. In the case of UCCSD, since it is an expansion in the coupling strength, at weak coupling the procedure is more reliable, and while there are substantial finite-size effects, the extrapolation produces a result within error bars of AFQMC and, in general, agreement with DMET.

\section{FREQUENCY AND MOMENTUM DEPENDENCE}

Next, we discuss single-particle finite-temperature properties. All finite-temperature algorithms discussed in this work are based on approximations of the single-particle self-energy. We show three characteristic plots for this quantity: Figure 15 shows the imaginary part of the local self-energy as a function of Matsubara frequency, Fig. 16 shows the dependence of the real part of the lowest Matsubara frequency on $k$ space, and Fig. 17 shows the frequency dependence of the imaginary part of the selfenergy for a specific momentum. Any discrepancy in the energy or double occupancy is the result of discrepancies in the single-particle self-energy.

The data shown in Fig. 15 are obtained for weak interaction strength $U / t=2$ and for a density $n=0.3$.

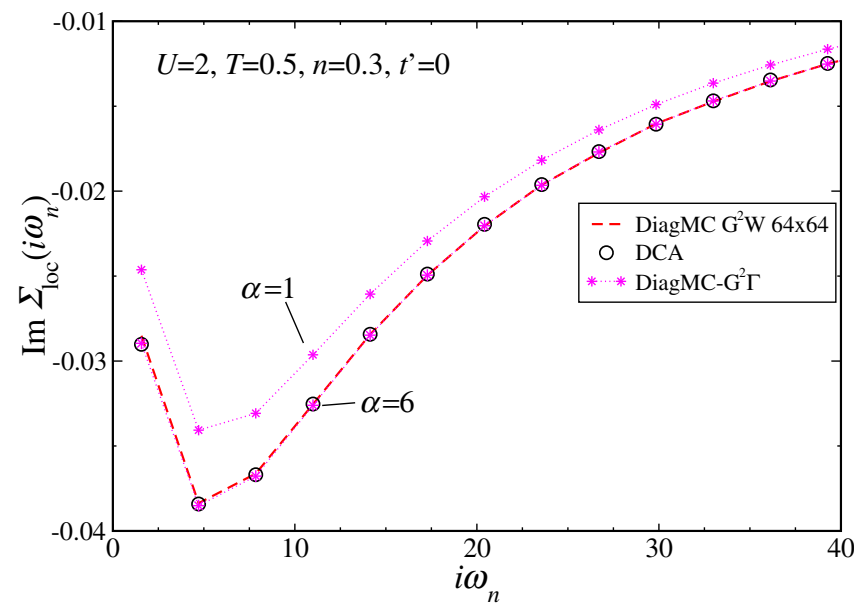

FIG. 15. Imaginary part of the local self-energy, $\operatorname{Im} \Sigma_{\text {loc }}\left(\mathrm{i} \omega_{\mathrm{n}}\right)$ at $U / t=2, T / t=0.5$ and $n=0.3$ from DCA and DiagMC. In the case of DiagMC- $G^{2} \Gamma$, we label $\alpha$, the series order from Eq. (7).

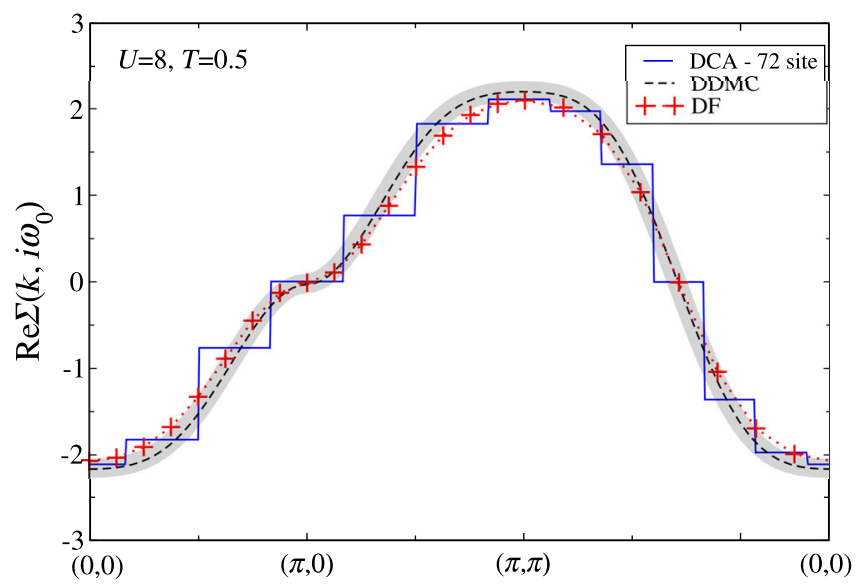

FIG. 16. Comparison of the real part of the self-energy, $\operatorname{Re} \Sigma\left(k, i \omega_{0}\right)$, at the lowest Matsubara frequency $i \omega_{0}$, obtained from DF (red) compared to 72-site DCA calculations (black) plotted as a function of momentum $k$, throughout the Brillouin zone for $n=1.0, U / t=8, T / t=0.5$. The dual fermion and DCA self-energies are plotted as step functions. Also included are interpolated results obtained by diagrammatic determinantal Monte Carlo (DDMC) [120,140,142,143] (dashed black) with a gray shading to indicate the level of uncertainty.

In this metallic regime, self-energies are small. Black circles denote the imaginary part of the local self-energy from an $N_{c}=20$ DCA calculation, which for these parameters shows essentially no finite-size effects. The data agree perfectly with DiagMC- $G^{2} W$ data shown as red dashed lines, and convergence of the DiagMC- $G^{2} \Gamma$ method to the result of the other two methods (stars, magenta dotted line) is observed as a function of expansion order $\alpha$. This agreement implies that the local physics is captured well by all three algorithms.

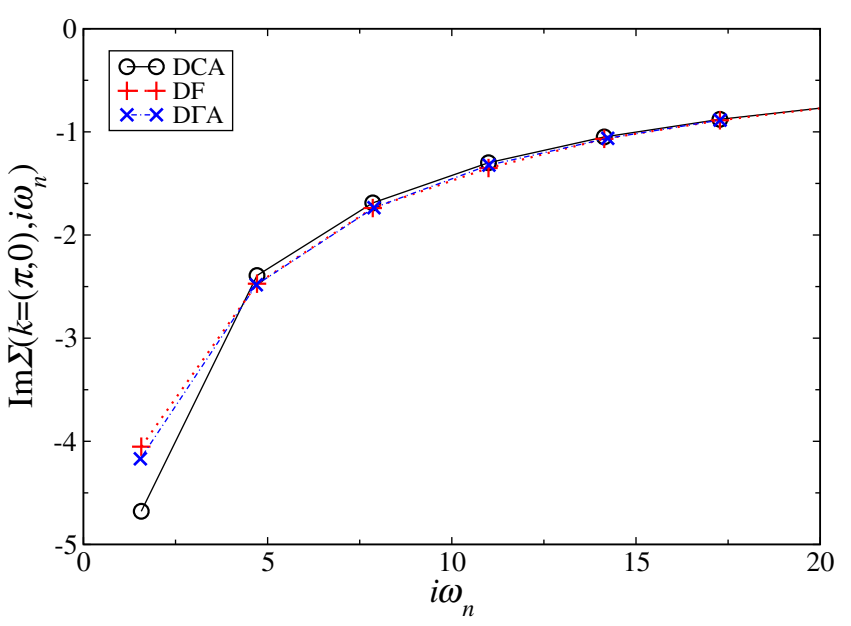

FIG. 17. Comparison of the frequency dependence of the imaginary part of the self-energy, $\operatorname{Im} \Sigma\left(k, i \omega_{n}\right)$, at fixed $k=$ $(\pi, 0)$ obtained from DF (red) compared to 72-site DCA calculations (black) plotted for $n=1.0, U / t=8, T / t=0.5$. Also shown are results from the dynamical vertex approximation DГA (blue) [124,144]. 
In Fig. 16, we examine momentum-dependent data. We show a path of $\left(k_{x}, k_{y}\right)$ through the Brillouin zone and plot the real part of the self-energy at the lowest Matsubara frequency at $U / t=8, \beta=2$, and $n=1$. DiagMC data are not available in this regime, but for comparison, we plot large DCA cluster results $\left(N_{c}=72\right)$ and results from continuous-time lattice Monte Carlo simulations (see Refs. [120,140,142,143]). The DCA approximation (blue lines) produces a stepdiscretized self-energy, which is in approximate agreement with the momentum dependence from other techniques. Discrepancies between the approximate DF method and the (essentially converged) DDMC method are visible but within the uncertainty of the DDMC comparison data. Any discrepancies are expected to rapidly disappear at higher Matsubara frequencies, which can be seen in a comparison of the imaginary part of the self-energy (Fig. 17) of DF and DCA at fixed momentum $k=(\pi, 0)$. For comparison or verification purposes, we include results from the dynamical vertex approximation (DГA, see Refs. [124,144,145]), which, in a spirit similar to DF, solves the model in an expansion of two-particle vertex functions. We find that the results from $\mathrm{DF}$ and $D \Gamma A$ are consistent.

\section{TABLES FOR GROUND-STATE PROPERTIES}

We conclude the discussion of our results with a list of the thermodynamic-limit estimates for the half-filled $t^{\prime}=0$ case. Table II shows a list of energies in the thermodynamic limit, obtained from AFQMC, DMRG, DMET, and FN. The MRPHF and UCCSD values presented show the value for the largest finite-size system studied. The uncertainties presented are the best uncertainties available within each algorithm and do not contain an assessment of systematic errors (e.g., fixed node or truncation in expansion order). We see that for much of phase space, errors are a few times $10^{-4} t$, and values between the techniques are remarkably consistent.

Table III shows the double occupancy for the same values. Relative errors for the double occupancy are of the same magnitude as for the total energy, and values in the thermodynamic limit are consistent within error bars. Tables IV and V present ground-state energies for the densities $n=0.8$ and 0.875 for values of $U / t$ considered in this work. The full table of values for the data presented in this paper is available online (see Ref. [46]).

Not all quantities are as consistent as the energies. This is especially true for order parameters and correlation functions, where discrepancies outside of error bars are present. Presumably, many competing phases exist in a narrow energy window near the ground state, and the most favorable state found in each method will depend on details of the finite-size system and the approximation. Table VI shows the comparison between the magnetization that DMET observes and the magnetization found in AFQMC for the full range of $U / t$ at half filling. At weak interaction strength, DMET finds a larger polarization than AFQMC even though the energies agree to all significant

TABLE II. Zero-temperature energy and uncertainty for $n=1, T=0$, for a range of interaction strengths $U$, obtained from AFQMC, DMET, DMRG, FN, MRPHF, and UCCSD. Where extrapolations to the TL are not available, finite-size geometries are listed in lieu of uncertainties. UCCSDTQ* data estimate higher-order corrections by including triples from a $[6 \times 6]$ and quadruples from a [4 $\times 4]$. UCCSD data for $U / t>4$ provide nearly converged energy estimates with respect to system size.

\begin{tabular}{|c|c|c|c|c|c|c|c|c|c|c|}
\hline \multirow{2}{*}{$\frac{\mathrm{U}}{\mathrm{AFQMC}}$} & \multicolumn{2}{|c|}{2} & \multicolumn{2}{|c|}{4} & \multicolumn{2}{|c|}{6} & \multicolumn{2}{|c|}{8} & \multicolumn{2}{|c|}{12} \\
\hline & -1.1763 & 0.0002 & -0.8603 & 0.0002 & -0.6568 & 0.0003 & -0.5247 & 0.0002 & -0.3693 & 0.0002 \\
\hline DMET & -1.1764 & 0.0003 & -0.8604 & 0.0003 & -0.6562 & 0.0005 & -0.5234 & 0.0010 & -0.3685 & 0.0010 \\
\hline DMRG & -1.176 & 0.001 & -0.8605 & 0.0005 & -0.6565 & 0.0001 & -0.5241 & 0.0001 & -0.3689 & 0.0001 \\
\hline FN & -1.175 & 0.001 & -0.8575 & 0.0003 & -0.6551 & 0.0001 & -0.52315 & 0.00005 & -0.36835 & 0.00005 \\
\hline MRPHF & -1.1628 & {$[8 \times 8]$} & -0.8554 & {$[8 \times 8]$} & -0.6512 & {$[8 \times 8]$} & -0.5169 & {$[8 \times 8]$} & -0.3626 & {$[8 \times 8]$} \\
\hline UCCSD & -1.1735 & 0.0004 & -0.8546 & {$[14 \times 14]$} & -0.6510 & {$[10 \times 10]$} & -0.5191 & {$[10 \times 10]$} & -0.3647 & {$[10 \times 10]$} \\
\hline UCCSDTQ* & -1.1749 & $\ldots$ & -0.8610 & $\ldots$ & -0.6582 & $\cdots$ & -0.5255 & $\cdots$ & -0.3696 & $\cdots$ \\
\hline
\end{tabular}

TABLE III. Zero-temperature double occupancy and uncertainty for $n=1$, for a range of interaction strengths $U$, obtained from AFQMC, DMET, DMRG, FN, MRPHF, and UCCSD. Where extrapolations to the TL are not available, finite-size geometries are listed in lieu of uncertainties.

\begin{tabular}{|c|c|c|c|c|c|c|c|c|c|c|}
\hline \multirow{2}{*}{$\frac{\mathrm{U}}{\mathrm{AFQMC}}$} & \multicolumn{2}{|c|}{2} & \multicolumn{2}{|c|}{4} & \multicolumn{2}{|c|}{6} & \multicolumn{2}{|c|}{8} & \multicolumn{2}{|c|}{12} \\
\hline & 0.1923 & 0.0003 & 0.1262 & 0.0002 & 0.0810 & 0.0001 & 0.0540 & 0.0001 & 0.0278 & 0.0001 \\
\hline DMET & 0.1913 & 0.0004 & 0.1261 & 0.0001 & 0.08095 & 0.00004 & 0.05398 & 0.00007 & 0.02780 & 0.00003 \\
\hline DMRG & 0.188 & 0.001 & 0.126 & 0.001 & 0.0809 & 0.0003 & 0.0539 & 0.0001 & 0.0278 & 0.0001 \\
\hline $\mathrm{FN}$ & 0.198 & 0.001 & 0.125 & 0.001 & 0.0803 & 0.0002 & 0.0535 & 0.0001 & 0.0278 & 0.0002 \\
\hline MRPHF & 0.1824 & {$[8 \times 8]$} & 0.1262 & {$[8 \times 8]$} & 0.0818 & {$[8 \times 8]$} & 0.0544 & {$[8 \times 8]$} & 0.0275 & {$[8 \times 8]$} \\
\hline UCCSD & 0.194 & 0.002 & 0.1268 & {$[12 \times 12]$} & 0.0807 & {$[10 \times 10]$} & 0.0537 & {$[10 \times 10]$} & 0.0276 & {$[10 \times 10]$} \\
\hline
\end{tabular}


TABLE IV. Zero-temperature energy and uncertainty for $n=0.8, T=0$, for a range of interaction strengths $U$, obtained from AFQMC (constrained path), DMET, DMRG, FN, MRPHF, and UCCSD. Where extrapolations to the TL are not available, finite-size geometries are listed in lieu of uncertainties.

\begin{tabular}{|c|c|c|c|c|c|c|c|c|}
\hline \multicolumn{9}{|c|}{$n=0.8$} \\
\hline \multirow{2}{*}{$\frac{\mathrm{U}}{\mathrm{AFQMC}}$} & \multicolumn{2}{|c|}{2} & \multicolumn{2}{|c|}{4} & \multicolumn{2}{|c|}{6} & \multicolumn{2}{|c|}{8} \\
\hline & -1.306 & 0.002 & -1.110 & 0.003 & $\cdots$ & $\ldots$ & $\cdots$ & $\cdots$ \\
\hline DMET & -1.3062 & 0.0004 & -1.108 & 0.002 & -0.977 & 0.004 & -0.88 & 0.03 \\
\hline DMRG & $\cdots$ & $\cdots$ & -1.104 & 0.0014 & $\cdots$ & $\cdots$ & $\cdots$ & $\cdots$ \\
\hline $\mathrm{FN}$ & -1.3044 & 0.0007 & -1.1032 & 0.0007 & -0.967 & 0.001 & -0.877 & 0.001 \\
\hline MRPHF & -1.2931 & {$[10 \times 4]$} & -1.0910 & {$[10 \times 4]$} & -0.9454 & {$[10 \times 4]$} & -0.8415 & {$[10 \times 4]$} \\
\hline UCCSD & -1.3065 & {$[10 \times 16]$} & -1.0868 & {$[10 \times 16]$} & -0.9300 & {$[10 \times 16]$} & -0.8233 & {$[10 \times 16]$} \\
\hline UCCSDT* & -1.3078 & {$[10 \times 4]$} & -1.0981 & {$[10 \times 4]$} & -0.9607 & {$[10 \times 4]$} & -0.8641 & {$[10 \times 4]$} \\
\hline
\end{tabular}

TABLE V. Zero-temperature energy and uncertainty for $n=0.875, T=0$, for a range of interaction strengths $U$, obtained from AFQMC (constrained path), DMET, DMRG, FN, MRPHF, and UCCSD. Where extrapolations to the $\mathrm{TL}$ are not available, finite-size geometries are listed in lieu of uncertainties.

\begin{tabular}{|c|c|c|c|c|c|c|c|c|}
\hline \multicolumn{9}{|c|}{$n=0.875$} \\
\hline \multirow{2}{*}{$\frac{\mathrm{U}}{\mathrm{AFQMC}}$} & \multicolumn{2}{|c|}{2} & \multicolumn{2}{|c|}{4} & \multicolumn{2}{|c|}{6} & \multicolumn{2}{|c|}{8} \\
\hline & $\cdots$ & $\cdots$ & -1.026 & 0.001 & $\cdots$ & $\cdots$ & -0.766 & 0.001 \\
\hline DMET & -1.2721 & 0.0006 & -1.031 & 0.003 & -0.863 & 0.013 & $-0.749^{\mathrm{a}}$ & 0.007 \\
\hline DMRG & $\ldots$ & $\ldots$ & -1.028 & {$[6 \times \infty]$} & $\cdots$ & $\ldots$ & -0.759 & 0.004 \\
\hline $\mathrm{FN}$ & -1.270 & 0.002 & -1.0225 & 0.0015 & -0.854 & 0.002 & -0.749 & 0.002 \\
\hline MRPHF & -1.2855 & {$[16 \times 4]$} & -1.0195 & {$[16 \times 4]$} & -0.8318 & {$[16 \times 4]$} & -0.7094 & {$[16 \times 4]$} \\
\hline UCCSD & -1.2667 & {$[16 \times 12]$} & -1.0093 & {$[16 \times 12]$} & -0.8298 & {$[16 \times 12]$} & -0.7123 & {$[16 \times 12]$} \\
\hline UCCSDT* & -1.2681 & {$[16 \times 4]$} & -1.0253 & {$[16 \times 4]$} & -0.8570 & {$[16 \times 4]$} & -0.7434 & {$[16 \times 4]$} \\
\hline
\end{tabular}

${ }^{a}$ Due to strong spatial inhomogeneity observed at this filling, the TL extrapolated number in the table is not meaningful, as different cluster shapes show different orders. Out of the clusters used here, the $8 \times 2$ impurity cluster is likely the best estimate with $\mathrm{E} / \mathrm{t}=-0.755( \pm 0.007)$. An exhaustive study of the DMET cluster size dependence at this filling will be carried out in the future.

TABLE VI. Comparison of magnetization data from DMET and AFQMC at $n=1$.

\begin{tabular}{|c|c|c|c|c|c|c|c|c|}
\hline \multirow{2}{*}{$\frac{\mathrm{U}}{\text { Technique }}$} & \multicolumn{2}{|c|}{2} & \multicolumn{2}{|c|}{4} & \multicolumn{2}{|c|}{6} & \multicolumn{2}{|c|}{8} \\
\hline & $m$ & $\delta m$ & $m$ & $\delta m$ & $m$ & $\delta m$ & $m$ & $\delta m$ \\
\hline AFQMC & 0.094 & 0.004 & 0.236 & 0.001 & 0.280 & 0.005 & 0.26 & 0.03 \\
\hline DMET & 0.133 & 0.005 & 0.252 & 0.009 & 0.299 & 0.012 & 0.318 & 0.013 \\
\hline
\end{tabular}

digits, as a result of DMET finite-size scaling from small clusters. At large interaction strength, AFQMC gives a polarization with large statistical fluctuation despite very accurate energies. Similar behavior (not shown here) is apparent for other variables, e.g., the $d$-wave order parameter or the stripe geometry observed at $U / t=8$ and $n=0.875$.

\section{CONCLUSIONS}

In this paper, we have presented a detailed examination of results for static and dynamic properties of the twodimensional Hubbard model at correlation strengths ranging from weak to intermediate to strong coupling, and at various carrier concentrations, obtained using state-of-theart numerical methods. We believe the results are useful for two reasons. First, the two-dimensional Hubbard model is one of the paradigm models of quantum condensed-matter theory, and it is therefore important to determine, as reliably as possible, the state of our knowledge about it. Second, solving the grand-challenge problem of determining the physics of interacting many-electron systems will require numerics, and as no one technique is likely to provide solutions in all regimes or for all quantities of physical interest, it is important to develop tools for assessing the strengths and weaknesses of different approaches.

We argue that the only quantities that can meaningfully be compared between different approaches are estimates, 
with error bars, for the thermodynamic-limit values of observables, including local operators such as the energy, double occupancy, density (or chemical potential), and magnetization, as well as correlation functions such as the electron self-energy. We restrict our attention to methods and regimes for which large enough systems can be studied such that reasonable extrapolations to the thermodynamic limit can be performed. Care is required in performing the extrapolations, and we have found it useful to present both the extrapolated results and (in most cases) the finite-size data that led to the extrapolation.

Comparison of results obtained from different methods shows that the ground-state properties of a substantial part of the Hubbard model phase space are now under numerical control (see, e.g., Figs. 4 and 12). Moreover, where there is agreement on the ground-state properties, the nonzero temperature methods appear to connect smoothly to the ground state as the temperature is decreased, although a quantitative extrapolation to $T=0$ is not yet available. The most substantial uncertainties exist at intermediate correlations (e.g., $U / t \approx 4 \rightarrow 8$ ) and at dopings near but not equal to the half-filling value $n=1$. In this intermediate coupling-near half-filling regime, several physically different states seem to have very similar energies, and small effects can favor the choice of one state over the other, leading to substantial uncertainties in physical quantities. Also, the issues associated with fermion sign problems seem to be most severe. Interestingly, it is this regime that is of most physical interest in connection with high- $T_{c}$ superconductivity in the copper-oxide materials.

Where two or more methods produce results that agree within reasonable error bars, we take the result to be established and appropriate for use as a benchmark. Tables of our benchmark results are contained in Ref. [46] and made available online. We expect that these results will be useful in validating new methods or new implementations of existing methods.

Turning now to prospects and open questions, we first observe that all of the methods we have considered to date have difficulty in the physically interesting intermediate coupling, near the half-filling regime. Development of new methods, or improvement of existing methods to deal with this regime, is urgently needed. Furthermore, we remark that our understanding of dynamical correlation functions, even ones as simple as the electron Green's function, is much less advanced than our understanding of ground-state properties and simple expectation values. Finally, we observe that the process of producing this paper, in particular, the confrontation of each method with the body of related work produced by other methods, led in several cases to substantial improvements in algorithm and error analysis. We suggest that as quantitative numerics in the many-electron field continues to evolve, intercomparison of different methods, leading to benchmarking on important model problems, will significantly advance the field.

\section{ACKNOWLEDGMENTS}

We acknowledge the Simons Foundation for funding. The work at Rice University was supported by Grant No. NSF-CHE-1462434. G. K. C. acknowledges funding from the U.S. Department of Energy (DOE) for the development of the DMET method through Grant No. DESC0010530, and for its application to the Hubbard model and superconductivity through Grant No. DE-SC0008624. F. B. and L.F. T. acknowledge support from PRIN 2010_2010LLKJBX. S. Z. and H. S. acknowledge support from the National Science Foundation Grant No. DMR1409510 for AFQMC method development. M. Q. was also supported by DOE Grant No. DE-SC0008627, and A. E. A. by DOE Grant No. ER 46932. Y. D. and X. W. L. acknowledge support from NSFC Grant No. 11275185. The AFQMC calculations were carried out at the Oak Ridge Leadership Computing Facility at the Oak Ridge National Laboratory, and at the computational facilities at the College of William and Mary.

[1] J. Hubbard, Electron Correlations in Narrow Energy Bands, Proc. R. Soc. A 276, 238 (1963).

[2] M. C. Gutzwiller, Effect of Correlation on the Ferromagnetism of Transition Metals, Phys. Rev. Lett. 10, 159 (1963).

[3] J. Kanamori, Electron Correlation and Ferromagnetism of Transition Metals, Prog. Theor. Phys. 30, 275 (1963).

[4] E. H. Lieb and F. Y. Wu, Absence of Mott Transition in an Exact Solution of the Short-Range, One-Band Model in One Dimension, Phys. Rev. Lett. 20, 1445 (1968).

[5] W. Metzner and D. Vollhardt, Correlated Lattice Fermions in $\mathrm{d}=\infty$ Dimensions, Phys. Rev. Lett. 62, 324 (1989).

[6] E. Müller-Hartmann, Correlated Fermions on a Lattice in High Dimensions, Z. Phys. B 74, 507 (1989).

[7] J. Oitmaa, C. Hamer, and W. Zheng, Series Expansion Methods for Strongly Interacting Lattice Models (Cambridge University Press, Cambridge, England, 2006).

[8] R. Valent, J. Stolze, and P. J. Hirschfeld, Lower Bounds for the Ground-State Energies of the Two-Dimensional Hubbard and t-J Models, Phys. Rev. B 43, 13743 (1991).

[9] R. Valent, C. Gros, P. Hirschfeld, and W. Stephan, Rigorous Bounds for Ground-State Properties of Correlated Fermi Systems, Phys. Rev. B 44, 13203 (1991).

[10] M. Y. Kagan and A. V. Chubukov, The Enhancement of a Superfluid Transition Temperature in a Polarised FermiGas with Repulsion, JETP Lett. 50, 517 (1989).

[11] M. A. Baranov and M. Y. Kagan, D-Wave Pairing in the Two-Dimensional Hubbard Model with Low Filling, Z. Phys. B 86, 237 (1992).

[12] A. V. Chubukov and J. P. Lu, Pairing Instabilities in the Two-Dimensional Hubbard Model, Phys. Rev. B 46, 11163 (1992).

[13] A. V. Chubukov, Kohn-Luttinger Effect and the Instability of a Two-Dimensional Repulsive Fermi Liquid at $\mathrm{T}=0$, Phys. Rev. B 48, 1097 (1993). 
[14] R. Hlubina, Phase Diagram of the Weak-Coupling TwoDimensional $t-t^{\prime}$ Hubbard Model at Low and Intermediate Electron Density, Phys. Rev. B 59, 9600 (1999).

[15] D. Zanchi and H. J. Schulz, Superconducting Instabilities of the Non-half-filled Hubbard Model in Two Dimensions, Phys. Rev. B 54, 9509 (1996).

[16] C. J. Halboth and W. Metzner, d-wave Superconductivity and Pomeranchuk Instability in the Two-Dimensional Hubbard Model, Phys. Rev. Lett. 85, 5162 (2000).

[17] P. W. Anderson, New Approach to the Theory of Superexchange Interactions, Phys. Rev. 115, 2 (1959).

[18] Y. Nagaoka, Ferromagnetism in a Narrow, Almost HalfFilled s Band, Phys. Rev. 147, 392 (1966).

[19] D Scalapino, Numerical Studies of the 2D Hubbard Model, in Handbook of High-Temperature Superconductivity, edited by J. Schrieffer and J. Brooks (Springer, New York, 2007), pp. 495-526.

[20] J. Gukelberger, L. Huang, and P. Werner, On the Dangers of Partial Diagrammatic Summations: Benchmarks for the Two-Dimensional Hubbard Model in the Weak-Coupling Regime, Phys. Rev. B 91, 235114 (2015).

[21] S. Zhang, Auxiliary-Field Quantum Monte Carlo for Correlated Electron Systems, in Emergent Phenomena in Correlated Matter Modeling and Simulation, edited by E. Pavarini, E. Koch, and U. Schollwöck (Verlag des Forschungszentrums Jülich, 2013), Vol. 3.

[22] H. Shi and S. Zhang, Infinite Variance in Fermion Quantum Monte Carlo Calculations, arXiv:1511.04084.

[23] H. Shi and S. Zhang, Symmetry in Auxiliary-Field Quantum Monte Carlo Calculations, Phys. Rev. B 88, 125132 (2013).

[24] N. V. Prokof'ev and B. V. Svistunov, Polaron Problem by Diagrammatic Quantum Monte Carlo, Phys. Rev. Lett. 81, 2514 (1998).

[25] A. Mishchenko, N. Prokofev, A. Sakamoto, and B. Svistunov, Diagrammatic Quantum Monte Carlo Study of the Fröhlich Polaron, Phys. Rev. B 62, 6317 (2000).

[26] K. Van Houcke, E. Kozik, N. Prokof'ev, and B. Svistunov, Diagrammatic Monte Carlo, Phys. Procedia 6, 95 (2010).

[27] A. N. Rubtsov, M. I. Katsnelson, and A. I. Lichtenstein, Dual Fermion Approach to Nonlocal Correlations in the Hubbard Model, Phys. Rev. B 77, 033101 (2008).

[28] G. Knizia and G. Kin Lic Chan, Density Matrix Embedding: A Simple Alternative to Dynamical Mean-Field Theory, Phys. Rev. Lett. 109, 186404 (2012).

[29] G. Knizia and G. Kin Lic Chan, Density Matrix Embedding: A Strong-Coupling Quantum Embedding Theory, J. Chem. Theory Comput. 9, 1428 (2013).

[30] S. R. White, Density Matrix Formulation for Quantum Renormalization Groups, Phys. Rev. Lett. 69, 2863 (1992).

[31] S. R. White, Density-Matrix Algorithms for Quantum Renormalization Groups, Phys. Rev. B 48, 10345 (1993).

[32] T. Maier, M. Jarrell, T. Pruschke, and M. H. Hettler, Quantum Cluster Theories, Rev. Mod. Phys. 77, 1027 (2005).

[33] C. Gros, Superconductivity in Correlated Wave Functions, Phys. Rev. B 38, 931(R) (1988).

[34] C. Gros, Physics of Projected Wavefunctions, Ann. Phys. (N.Y.) 189, 53 (1989).
[35] H. Yokoyama and H. Shiba, Variational Monte-Carlo Studies of Hubbard Model. I, J. Phys. Soc. Jpn. 56, 1490 (1987).

[36] H. Yokoyama and H. Shiba, Variational Monte-Carlo Studies of Hubbard Model. II, J. Phys. Soc. Jpn. 56, 3582 (1987).

[37] L. F. Tocchio, F. Becca, A. Parola, and S. Sorella, Role of Backflow Correlations for the Nonmagnetic Phase of the $t-t^{\prime}$ Hubbard Model, Phys. Rev. B 78, 041101(R) (2008).

[38] N. Trivedi and D. M. Ceperley, Ground-State Correlations of Quantum Antiferromagnets: A Green-Function Monte Carlo Study, Phys. Rev. B 41, 4552 (1990).

[39] D. F. B. ten Haaf, H. J. M. van Bemmel, J. M. J. van Leeuwen, W. van Saarloos, and D. M. Ceperley, Proof for an Upper Bound in Fixed-Node Monte Carlo for Lattice Fermions, Phys. Rev. B 51, 13039 (1995).

[40] J. Paldus and X. Li, A Critical Assessment of Coupled Cluster Method in Quantum Chemistry, Adv. Chem. Phys. 110, 1 (1999).

[41] R. J. Bartlett and M. Musiał, Coupled-Cluster Theory in Quantum Chemistry, Rev. Mod. Phys. 79, 291 (2007).

[42] I. Shavitt and R. J. Bartlett, Many-Body Methods in Chemistry and Physics (Cambridge University Press, New York, 2009).

[43] R. Rodríguez-Guzmán, K. W. Schmid, C. A. JiménezHoyos, and G. E. Scuseria, Symmetry-Projected Variational Approaches for Ground and Excited States of the One-Dimensional Hubbard Model, Phys. Rev. B 85, 245130 (2012).

[44] R. Rodríguez-Guzmán, C. A. Jiménez-Hoyos, R. Schutski, and G. E. Scuseria, Multireference Symmetry-Projected Variational Approaches for Ground and Excited States of the One-Dimensional Hubbard Model, Phys. Rev. B 87, 235129 (2013).

[45] J. P. F. LeBlanc et al., Github Repository, doi:10.5281/ zenodo.34433 (2015).

[46] See Supplemental Material at http://link.aps.org/ supplemental/10.1103/PhysRevX.5.041041 for complete data sets.

[47] R. Bulla, T. A. Costi, and T. Pruschke, Numerical Renormalization Group Method for Quantum Impurity Systems, Rev. Mod. Phys. 80, 395 (2008).

[48] E. Dagotto, Correlated Electrons in High-Temperature Superconductors, Rev. Mod. Phys. 66, 763 (1994).

[49] M van Schilfgaarde, T. Kotani, and S. Faleev, Quasiparticle Self-Consistent GW Theory, Phys. Rev. Lett. 96, 226402 (2006).

[50] M. S. Hybertsen and S. G. Louie, Electron Correlation in Semiconductors and Insulators: Band Gaps and Quasiparticle Energies, Phys. Rev. B 34, 5390 (1986).

[51] R. Blankenbecler, D. J. Scalapino, and R. L. Sugar, Monte Carlo Calculations of Coupled Boson-Fermion Systems. I, Phys. Rev. D 24, 2278 (1981).

[52] G. Sugiyama and S. E. Koonin, Auxiliary Field MonteCarlo for Quantum Many-Body Ground States, Ann. Phys. (N.Y.) 168, 1 (1986).

[53] S. R. White, D. J. Scalapino, R. L. Sugar, E. Y. Loh, J. E. Gubernatis, and R. T. Scalettar, Numerical Study of the Two-Dimensional Hubbard Model, Phys. Rev. B 40, 506 (1989). 
[54] H. Shi, S. Chiesa, and S. Zhang, Ground-State Properties of Strongly Interacting Fermi Gases in Two Dimensions, Phys. Rev. A 92, 033603 (2015).

[55] S. Zhang and H. Krakauer, Quantum Monte Carlo Method Using Phase-Free Random Walks with Slater Determinants, Phys. Rev. Lett. 90, 136401 (2003).

[56] E. Y. Loh, J. E. Gubernatis, R. T. Scalettar, S. R. White, D. J. Scalapino, and R. L. Sugar, Sign Problem in the Numerical Simulation of Many-Electron Systems, Phys. Rev. B 41, 9301 (1990).

[57] J. E. Hirsch, Two-Dimensional Hubbard Model: Numerical Simulation Study, Phys. Rev. B 31, 4403 (1985).

[58] S. Zhang, J. Carlson, and J. E. Gubernatis, Constrained Path Monte Carlo Method for Fermion Ground States, Phys. Rev. B 55, 7464 (1997).

[59] H. Nguyen, H. Shi, J. Xu, and S. Zhang, CPMC-Lab: A Matlab Package for Constrained Path Monte Carlo Calculations, Comput. Phys. Commun. 185, 3344 (2014).

[60] C. Lin, F. H. Zong, and D. M. Ceperley, Twist-Averaged Boundary Conditions in Continuum Quantum Monte Carlo Algorithms, Phys. Rev. E 64, 016702 (2001).

[61] C.-C. Chang and S. Zhang, Spatially Inhomogeneous Phase in the Two-Dimensional Repulsive Hubbard Model, Phys. Rev. B 78, 165101 (2008).

[62] C.-C. Chang and S. Zhang, Spin and Charge Order in the Doped Hubbard Model: Long-Wavelength Collective Modes, Phys. Rev. Lett. 104, 116402 (2010).

[63] D. A. Huse, Ground-State Staggered Magnetization of Two-Dimensional Quantum Heisenberg Antiferromagnets, Phys. Rev. B 37, 2380 (1988).

[64] H. Neuberger and T. Ziman, Finite-Size Effects in Heisenberg Antiferromagnets, Phys. Rev. B 39, 2608 (1989).

[65] D. S. Fisher, Universality, Low-Temperature Properties, and Finite-Size Scaling in Quantum Antiferromagnets, Phys. Rev. B 39, 11783 (1989).

[66] S. R. White and D. J. Scalapino, Density Matrix Renormalization Group Study of the Striped Phase in the 2D t-J Model, Phys. Rev. Lett. 80, 1272 (1998).

[67] H. Shi, C. A. Jiménez-Hoyos, R. Rodríguez-Guzmán, G. E. Scuseria, and S. Zhang, Symmetry-Projected Wave Functions in Quantum Monte Carlo Calculations, Phys. Rev. B 89, 125129 (2014).

[68] M. Capello, F. Becca, M. Fabrizio, S. Sorella, and E. Tosatti, Variational Description of Mott Insulators, Phys. Rev. Lett. 94, 026406 (2005).

[69] M. Capello, F. Becca, S. Yunoki, and S. Sorella, Unconventional Metal-Insulator Transition in Two Dimensions, Phys. Rev. B 73, 245116 (2006).

[70] F. Becca, M. Capone, and S. Sorella, Spatially Homogeneous Ground State of the Two-Dimensional Hubbard Model, Phys. Rev. B 62, 12700 (2000).

[71] L. F. Tocchio, F. Becca, and C. Gros, Backflow Correlations in the Hubbard Model: An Efficient Tool for the Study of the Metal-Insulator Transition and the Large-U Limit, Phys. Rev. B 83, 195138 (2011).

[72] A. Leprévost, O. Juillet, and R. Frésard, Intertwined Orders from Symmetry Projected Wavefunctions of Repulsively Interacting Fermi Gases in Optical Lattices, New J. Phys. 17, 103023 (2015).
[73] P. Ring and P. Schuck, The Nuclear Many-Body Problem (Springer-Verlag, Berlin, 1980).

[74] K. W. Schmid, On the Use of General Symmetry-Projected Hartree-Fock-Bogoliubov Configurations in Variational Approaches to the Nuclear Many-Body Problem, Prog. Part. Nucl. Phys. 52, 565 (2004).

[75] N. Tomita, Many-Body Wave Functions Approximated by the Superposition of Spin-Projected Nonorthogonal Slater Determinants in the Resonating Hartree-Fock Method, Phys. Rev. B 69, 045110 (2004).

[76] R. Rodríguez-Guzmán, C. A. Jiménez-Hoyos, and G. E. Scuseria, Variational Description of the Ground State of the Repulsive Two-Dimensional Hubbard Model in Terms of Nonorthogonal Symmetry-Projected Slater Determinants, Phys. Rev. B 90, 195110 (2014).

[77] T. Mizusaki and M. Imada, Quantum-Number Projection in the Path-Integral Renormalization Group Method, Phys. Rev. B 69, 125110 (2004).

[78] D. Tahara and M. Imada, Variational Monte Carlo Method Combined with Quantum-Number Projection and MultiVariable Optimization, J. Phys. Soc. Jpn. 77, 114701 (2008).

[79] C. A. Jiménez-Hoyos and G. E. Scuseria, Cluster-Based Mean-Field and Perturbative Description of Strongly Correlated Fermion Systems: Application to the Oneand Two-Dimensional Hubbard Model, Phys. Rev. B 92, 085101 (2015).

[80] P. Corboz, T. M. Rice, and M. Troyer, Competing States in the $t$-j Model: Uniform $d$-wave State versus Stripe State, Phys. Rev. Lett. 113, 046402 (2014).

[81] P. Corboz, S. R. White, G. Vidal, and M. Troyer, Stripes in the Two-Dimensional $t$-J Model with Infinite Projected Entangled-Pair States, Phys. Rev. B 84, 041108 (2011).

[82] F. Petit and M. Roger, Coupled-Cluster Method Applied to the Motion of a Single Hole in a Hubbard Antiferromagnet, Phys. Rev. B 49, 3453 (1994).

[83] R. F. Bishop, Y. Xian, and C. Zeng, A Microscopic Coupled-Cluster Treatment of Electronic Correlations in Hubbard Models, Int. J. Quantum Chem. 55, 181 (1995).

[84] Y. Asai and H. Katagiri, Coupled-Cluster Approach to Electron Correlations in the Two-Dimensional Hubbard Model, Phys. Rev. B 60, R13946 (1999).

[85] G. D. Purvis and R. J. Bartlett, A Full Coupled-Cluster Singles and Doubles Model-The Inclusion of Disconnected Triples, J. Chem. Phys. 76, 1910 (1982).

[86] G. E. Scuseria, A. Sc. Scheiner, T. J. Lee, J. E. Rice, and H. F. Schaeffer III, The Closed-Shell Coupled Cluster Single and Double (CCSD) Excitation Model for the Description of Electron Correlation: A Comparison with Configuration Interaction (CISD) Results, J. Chem. Phys. 86, 2881 (1987).

[87] J. Xue, C.-C. Cheng, E. J. Walter, and S. Zhang, Spin- and Charge-Density Waves in the Hartree-Fock Ground State of the Two-Dimensional Hubbard Model, J. Phys. Condens. Matter 23, 505601 (2011).

[88] N. C. Handy, J. A. Pople, M. Head-Gordon, K. Raghavachari, and G. W. Trucks, Size-Consistent Brueckner Theory Limited to Double Substitutions, Chem. Phys. Lett. 164, 185 (1989).

[89] M. J. Frisch et al., Gaussian Development Version, Revision H.21 (Gaussian Inc., Wallingford, CT, 2010). 
[90] M. Kállay, Z. Rolik, J. Csontos, I. Ladjánszki, L. Szegedy, B. Ladóczki, and G. Samu, www.mrcc.hu; See also, Z. Rolik, L. Szegedy, I. Ladjánszki, B. Ladóczki, and M. Kállay, An Efficient Linear-Scaling CCSD(T) Method Based on Local Natural Orbitals, J. Chem. Phys. 139, 094105 (2013).

[91] F. Verstraete and J. I. Cirac, Renormalization Algorithms for Quantum-Many Body Systems in Two and Higher Dimensions, arXiv:cond-mat/0407066.

[92] G. Vidal, Entanglement Renormalization, Phys. Rev. Lett. 99, 220405 (2007).

[93] S. R. White and A. L. Chernyshev, Neel Order in Square and Triangular Lattice Heisenberg Models, Phys. Rev. Lett. 99, 127004 (2007).

[94] E. M. Stoudenmire and S. R. White, Studying TwoDimensional Systems with the Density Matrix Renormalization Group, Annu. Rev. Condens. Matter Phys. 3, 111 (2012).

[95] A. W. Sandvik, Finite-Size Scaling of the Ground-State Parameters of the Two-Dimensional Heisenberg Model, Phys. Rev. B 56, 11678 (1997).

[96] I. Peschel, Special Review: Entanglement in Solvable Many-Particle Models, Braz. J. Phys. 42, 267 (2012).

[97] G. H. Booth and G. Kin-Lic Chan, Spectral Functions of Strongly Correlated Extended Systems via an Exact Quantum Embedding, Phys. Rev. B 91, 155107 (2015).

[98] I. W. Bulik, G. E. Scuseria, and J. Dukelsky, Density Matrix Embedding from Broken Symmetry Lattice Mean Fields, Phys. Rev. B 89, 035140 (2014).

[99] Q. Chen, G. H. Booth, S. Sharma, G. Knizia, and G. Kin Lic Chan, Intermediate and Spin-Liquid Phase of the HalfFilled Honeycomb Hubbard model, Phys. Rev. B 89, 165134 (2014).

[100] P.-G. de Gennes, Superconductivity of Metals and Alloys (Benjamin, New York, 1966).

[101] D. Yamaki, T. Ohsaku, H. Nagao, and K. Yamaguchi, Formulation of Unrestricted and Restricted HartreeFock-Bogoliubov Equations, Int. J. Quantum Chem. 96, 10 (2004).

[102] G. Kin-Lic Chan and M. Head-Gordon, Highly Correlated Calculations with a Polynomial Cost Algorithm: A Study of the Density Matrix Renormalization Group, J. Chem. Phys. 116, 4462 (2002).

[103] G. K. L. Chan, An Algorithm for Large Scale Density Matrix Renormalization Group Calculations, J. Chem. Phys. 120, 3172 (2004).

[104] G. Kin-Lic Chan and S. Sharma, The Density Matrix Renormalization Group in Quantum Chemistry, Annu. Rev. Phys. Chem. 62, 465 (2011).

[105] Ö. Legeza and G. Fáth, Accuracy of the Density-Matrix Renormalization-Group Method, Phys. Rev. B 53, 14349 (1996).

[106] B.-X. Zheng and G. Kin-Lic Chan, Ground-State Phase Diagram of the Square Lattice Hubbard Model from Density Matrix Embedding Theory, arXiv:1504.01784.

[107] M. H. Hettler, A. N. Tahvildar-Zadeh, M. Jarrell, T. Pruschke, and H. R. Krishnamurthy, Nonlocal Dynamical Correlations of Strongly Interacting Electron Systems, Phys. Rev. B 58, R7475 (1998).
[108] M. H. Hettler, M. Mukherjee, M. Jarrell, and H. R. Krishnamurthy, Dynamical Cluster Approximation: Nonlocal Dynamics of Correlated Electron Systems, Phys. Rev. B 61, 12739 (2000).

[109] A. Georges and G. Kotliar, Hubbard Model in Infinite Dimensions, Phys. Rev. B 45, 6479 (1992).

[110] A. Georges, G. Kotliar, W. Krauth, and M. J. Rozenberg, Dynamical Mean-Field Theory of Strongly Correlated Fermion Systems and the Limit of Infinite Dimensions, Rev. Mod. Phys. 68, 13 (1996).

[111] A. I. Lichtenstein and M. I. Katsnelson, Antiferromagnetism and d-wave Superconductivity in Cuprates: A Cluster Dynamical Mean-Field Theory, Phys. Rev. B 62, R9283 (2000).

[112] G. Kotliar, S. Y. Savrasov, G. Pálsson, and G. Biroli, Cellular Dynamical Mean Field Approach to Strongly Correlated Systems, Phys. Rev. Lett. 87, 186401 (2001).

[113] S. Fuchs, E. Gull, L. Pollet, E. Burovski, E. Kozik, T. Pruschke, and M. Troyer, Thermodynamics of the $3 D$ Hubbard Model on Approaching the Néel Transition, Phys. Rev. Lett. 106, 030401 (2011).

[114] J. P. F. LeBlanc and E. Gull, Equation of State of the Fermionic Two-Dimensional Hubbard Model, Phys. Rev. B 88, 155108 (2013).

[115] E. Gull, P. Werner, O. Parcollet, and M. Troyer, ContinuousTime Auxiliary-Field Monte Carlo for Quantum Impurity Models, Europhys. Lett. 82, 57003 (2008).

[116] E. Gull, A. J. Millis, A. I. Lichtenstein, A. N. Rubtsov, M. Troyer, and P. Werner, Continuous-Time Monte Carlo Methods for Quantum Impurity Models, Rev. Mod. Phys. 83, 349 (2011).

[117] E. Gull, P. Staar, S. Fuchs, P. Nukala, M. S. Summers, T. Pruschke, T. C. Schulthess, and T. Maier, Submatrix Updates for the Continuous-Time Auxiliary-Field Algorithm, Phys. Rev. B 83, 075122 (2011).

[118] B. Bauer, L. D. Carr, H. G. Evertz, A. Feiguin, J. Freire, S. Fuchs, L. Gamper, J. Gukelberger, E. Gull, S. Guertler et al., The ALPS Project Release 2.0: Open Source Software for Strongly Correlated Systems, J. Stat. Mech. Theor. Exp. (2011) P05001.

[119] E. Gull, P. Werner, S. Fuchs, B. Surer, T. Pruschke, and M. Troyer, Continuous-Time Quantum Monte Carlo Impurity Solvers, Comput. Phys. Commun. 182, 1078 (2011).

[120] A. N. Rubtsov, V. V. Savkin, and A. I. Lichtenstein, Continuous-Time Quantum Monte Carlo Method for Fermions, Phys. Rev. B 72, 035122 (2005).

[121] O. Parcollet, M. Ferrero, T. Ayral, H. Hafermann, I. Krivenko, L. Messio, and P. Seth, TRIQS: A Toolbox for Research on Interacting Quantum Systems, Comput. Phys. Commun. 196, 398 (2015).

[122] A. N. Rubtsov, M. I. Katsnelson, A. I. Lichtenstein, and A. Georges, Dual Fermion Approach to the Two-Dimensional Hubbard Model: Antiferromagnetic Fluctuations and Fermi Arcs, Phys. Rev. B 79, 045133 (2009).

[123] H. Hafermann, F. Lechermann, A. N. Rubtsov, M. I. Katsnelson, A. Georges, and A. I. Lichtenstein, Modern Theories of Many-Particle Systems in Condensed Matter Physics, in Modern Theories of Many-Particle Systems in Condensed Matter Physics, Lecture Notes in Physics, 
edited by D. C. Cabra, A. Honecker, and P. Pujol (Springer, Berlin-Heidelberg, 2012), Vol. 843, pp. 145-214.

[124] A. Toschi, A. A. Katanin, and K. Held, Dynamical Vertex Approximation: A Step Beyond Dynamical Mean-Field Theory, Phys. Rev. B 75, 045118 (2007).

[125] C. Taranto, S. Andergassen, J. Bauer, K. Held, A. Katanin, W. Metzner, G. Rohringer, and A. Toschi, From Infinite to Two Dimensions through the Functional Renormalization Group, Phys. Rev. Lett. 112, 196402 (2014).

[126] N. Wentzell, C. Taranto, A. Katanin, A. Toschi, and S. Andergassen, Correlated Starting Points for the Functional Renormalization Group, Phys. Rev. B 91, 045120 (2015).

[127] A. E. Antipov, E. Gull, and S. Kirchner, Critical Exponents of Strongly Correlated Fermion Systems from Diagrammatic Multiscale Methods, Phys. Rev. Lett. 112, 226401 (2014).

[128] J. Otsuki, H. Hafermann, and A. I. Lichtenstein, Superconductivity, Antiferromagnetism, and Phase Separation in the Two-Dimensional Hubbard Model: A Dual-Fermion Approach, Phys. Rev. B 90, 235132 (2014).

[129] A. E. Antipov, J. P. F. LeBlanc, and E. Gull, Opendf-An Implementation of the Dual Fermion Method for Strongly Correlated Systems, Phys. Procedia 68, 43 (2015).

[130] A. L. Fetter and J. D. Walecka, Quantum Theory of ManyParticle Systems (Dover Publications Inc., New York, 2003).

[131] N. Prokofev and B. Svistunov, Bold Diagrammatic Monte Carlo Technique: When the Sign Problem Is Welcome, Phys. Rev. Lett. 99, 250201 (2007).

[132] K. Van Houcke, F. Werner, E. Kozik, N. Prokofev, B. Svistunov, M. J. H. Ku, A. T. Sommer, L. W. Cheuk, A. Schirotzek, and M. W. Zwierlein, Feynman Diagrams versus Fermi-Gas Feynman Emulator, Nat. Phys. 8, 366 (2012).

[133] E. Gull, D. R. Reichman, and A. J. Millis, Bold-Line Diagrammatic Monte Carlo Method: General Formulation and Application to Expansion Around the Noncrossing Approximation, Phys. Rev. B 82, 075109 (2010).

[134] E. Kozik, K. Van Houcke, E. Gull, L. Pollet, N. Prokof'ev, B. Svistunov, and M. Troyer, Diagrammatic Monte Carlo for Correlated Fermions, Europhys. Lett. 90, 10004 (2010).
[135] S. A. Kulagin, N. Prokofev, O. A. Starykh, B. Svistunov, and C. N. Varney, Bold Diagrammatic Monte Carlo Method Applied to Fermionized Frustrated Spins, Phys. Rev. Lett. 110, 070601 (2013).

[136] S. Kulagin, N. Prokof'ev, O. A. Starykh, B. V. Svistunov, and C.N. Varney, Bold Diagrammatic Monte Carlo Technique for Frustrated Spin Systems, Phys. Rev. B 87, 024407 (2013).

[137] A. S. Mishchenko, N. Nagaosa, and N. Prokofev, Diagrammatic Monte Carlo Method for Many-Polaron Problems, Phys. Rev. Lett. 113, 166402 (2014).

[138] K. Van Houcke, F. Werner, N. Prokof'ev, and B. Svistunov, Bold Diagrammatic Monte Carlo for the Resonant Fermi Gas, arXiv:1305.3901.

[139] Y. Deng, E. Kozik, N. V. Prokof'ev, and B. V. Svistunov, Emergent BCS Regime of the Two-Dimensional Fermionic Hubbard Model: Ground-State Phase Diagram, Europhys. Lett. 110, 57001 (2015).

[140] E. Kozik, M. Ferrero, and A. Georges, Nonexistence of the Luttinger-Ward Functional and Misleading Convergence of Skeleton Diagrammatic Series for Hubbard-Like Models, Phys. Rev. Lett. 114, 156402 (2015).

[141] S. Sorella, Linearized Auxiliary Fields Monte Carlo Technique: Efficient Sampling of the Fermion Sign, Phys. Rev. B 84, 241110 (2011).

[142] E. Burovski, N. Prokof'ev, B. Svistunov, and M. Troyer, The Fermi-Hubbard Model at Unitarity, New J. Phys. 8, 153 (2006).

[143] E. Kozik, E. Burovski, V. W. Scarola, and M. Troyer, Néel Temperature and Thermodynamics of the Half-Filled ThreeDimensional Hubbard Model by Diagrammatic Determinant Monte Carlo, Phys. Rev. B 87, 205102 (2013).

[144] T. Schäfer, F. Geles, D. Rost, G. Rohringer, E. Arrigoni, K. Held, N. Blümer, M. Aichhorn, and A. Toschi, Fate of the False Mott-Hubbard Transition in Two Dimensions, Phys. Rev. B 91, 125109 (2015).

[145] T. Schaefer, A. Toschi, and K. Held, Dynamical Vertex Approximation for the Two-Dimensional Hubbard Model, J. Magn. Magn. Mater., doi: 10.1016/j.jmmm.2015.07.103 (2015). 